\title{
Pulse sequences and parallel imaging for high spatiotemporal resolution MRI at ultra-high field
}

Citation for published version (APA):

Poser, B. A., \& Setsompop, K. (2018). Pulse sequences and parallel imaging for high spatiotemporal resolution MRI at ultra-high field. Neuroimage, 168, 101-118. https://doi.org/10.1016/j.neuroimage.2017.04.006

Document status and date:

Published: 01/03/2018

DOI:

10.1016/j.neuroimage.2017.04.006

Document Version:

Publisher's PDF, also known as Version of record

Document license:

Taverne

Please check the document version of this publication:

- A submitted manuscript is the version of the article upon submission and before peer-review. There can be important differences between the submitted version and the official published version of record.

People interested in the research are advised to contact the author for the final version of the publication, or visit the DOI to the publisher's website.

- The final author version and the galley proof are versions of the publication after peer review.

- The final published version features the final layout of the paper including the volume, issue and page numbers.

Link to publication

\footnotetext{
General rights rights.

- You may freely distribute the URL identifying the publication in the public portal. please follow below link for the End User Agreement:

www.umlib.nl/taverne-license

Take down policy

If you believe that this document breaches copyright please contact us at:

repository@maastrichtuniversity.nl

providing details and we will investigate your claim.
}

Copyright and moral rights for the publications made accessible in the public portal are retained by the authors and/or other copyright owners and it is a condition of accessing publications that users recognise and abide by the legal requirements associated with these

- Users may download and print one copy of any publication from the public portal for the purpose of private study or research.

- You may not further distribute the material or use it for any profit-making activity or commercial gain

If the publication is distributed under the terms of Article $25 \mathrm{fa}$ of the Dutch Copyright Act, indicated by the "Taverne" license above, 


\title{
Pulse sequences and parallel imaging for high spatiotemporal resolution MRI at ultra-high field
}

\author{
Benedikt A. Poser ${ }^{\mathrm{a}, *, 1}$, Kawin Setsompop ${ }^{\mathrm{b}, \mathrm{c}, 1}$ \\ a Department of Cognitive Neuroscience, Faculty of Psychology and Neuroscience, Maastricht University, Maastricht, Netherlands \\ b Department of Radiology, Harvard Medical School, Boston, MA, USA \\ ${ }^{\mathrm{c}}$ Athinoula A. Martinos Center for Biomedical Imaging, Massachusetts General Hospital, Charlestown, MA, USA
}

\section{A R T I C L E I N F O}

\section{Keyword:}

Ultra-high field

Parallel imaging

CAIPIRINHA

Wave-CAIPI

Simultaneous multi-slice

BOLD fMRI

dw-EPI

Structural MRI

QSM

\begin{abstract}
A B S T R A C T
The SNR and CNR benefits of ultra-high field (UHF) have helped push the envelope of achievable spatial resolution in MRI. For applications based on susceptibility contrast where there is a large CNR gain, high quality sub-millimeter resolution imaging is now being routinely performed, particularly in fMRI and phase imaging/QSM. This has enabled the study of structure and function of very fine-scale structures in the brain. UHF has also helped push the spatial resolution of many other MRI applications as will be outlined in this review. However, this push in resolution comes at a cost of a large encoding burden leading to very lengthy scans. Developments in parallel imaging with controlled aliasing and the move away from 2D slice-by-slice imaging to much more SNR-efficient simultaneous multi-slice (SMS) and 3D acquisitions have helped address this issue. In particular, these developments have revolutionized the efficiency of UHF MRI to enable high spatiotemporal resolution imaging at an order of magnitude faster acquisition. In addition to describing the main approaches to these techniques, this review will also outline important key practical considerations in using these methods in practice. Furthermore, new RF pulse design to tackle the $\mathrm{B}_{1}{ }^{+}$and SAR issues of UHF and the increased SAR and power requirement of SMS RF pulses will also be touched upon. Finally, an outlook into new developments of smart encoding in more dimensions, particularly through using better temporal/acrosscontrast encoding and reconstruction will be described. Just as controlled aliasing fully exploits spatial encoding in parallel imaging to provide large multiplicative gains in accelerations, the complimentary use of these new approaches in temporal and across-contrast encoding are expected to provide exciting opportunities for further large gains in efficiency to further push the spatiotemporal resolution of MRI.
\end{abstract}

\section{Introduction}

Ultra-high field (UHF) MRI at $7 \mathrm{~T}$ and above, has brought about great opportunities for improving the spatial and temporal resolution, thanks to large increases in signal to noise ratio (SNR) that can be traded for smaller voxel volumes and/or reduced acquisition times by undersampling. Moreover, the change of transverse and longitudinal relaxation times, along with enhanced phase contrast at UHF result in altered image contrasts that benefit several applications of anatomical and functional MRI and give rise to emerging techniques that have been unfeasible at the commonly used clinical field strengths of $1.5 \mathrm{~T}$ and $3 \mathrm{~T}$.

The shortened $\mathrm{T}_{2}{ }^{*}$ for example favors applications based on susceptibility contrast: The super-linear increases in the contrast-tonoise (CNR) ratio in functional BOLD imaging (Yacoub et al., 2001) allow fMRI to the detail of cortical layers, susceptibility weighed imaging (SWI) proves useful in the imaging of small venous vessels where paramagnetic blood serves as the endogenous contrast agent (Haacke et al., 2009; Koopmans et al., 2008), and quantitative susceptibility mapping (QSM) allows the fine delineation of subcortical structures and depiction of iron concentrations contained within them (Duyn et al., 2007; Fukunaga et al., 2010). A further important feature at UHF is the longer $T_{1}$ longitudinal relaxation time (unlike $T_{2}$ and $T_{2}{ }^{*}$ which decrease), which in arterial spin labelling (ASL) increases the affordable post-labelling and readout periods because the labelled spins keep their magnetic tag for longer (Gardener et al., 2009; Golay and Petersen, 2006; Pfeuffer et al., 2002a). The amplification of blood inflow effects moreover provides vessel contrast in time-of-flight angiography (von Morze et al., 2007; Zwanenburg et al., 2008) where again the moving blood is the 'in-built' contrast and the administration

\footnotetext{
* Corresponding author.

E-mail address: benedikt.poser@maastrichtuniversity.nl (B.A. Poser).

${ }^{1}$ Authors contributed equally.
} 
of external contrast agents is not required.

This paper reviews the various neuroimaging applications in which the move towards UHF has played an important enabling role. In this context we focus on the significant acquisition challenges that have been brought about by the desire to image at ever higher spatial resolutions, and the paper provides an overview of the recent state-ofthe art parallel imaging techniques and the move away from 2D sliceby-slice imaging to much more SNR-efficient simultaneous multi-slice (SMS) and 3D acquisitions that address these challenges with drastic improvements in acquisition speed.

\section{Benefits of high field}

\section{$B O L D$ fMRI}

Functional imaging has been one of the main drivers behind early high field human imaging, and it may be noted that the first $7 \mathrm{~T}$ installations were at centers with a primary interest in human brain function. Local changes in blood flow and volume that accompany brain activation give rise to the BOLD signal via four MRI contrast mechanisms, whose field strength dependence lead to an overall superlinear BOLD increase with $\mathrm{B}_{0}$, and an increasingly stronger weighting towards the microvasculature close to the neuronal activations. For review on the hemodynamics and BOLD contrast mechanisms the reader is referred to (Norris, 2006) and references therein. The vast majority of fMRI was and still is performed with gradient-echo EPI and is hence predominantly $T_{2}{ }^{*}$ weighted which offers excellent sensitivity at high sampling rates and low RF power deposition. Early submillimeter resolution fMRI experiments at high field were demonstrated with FLASH type sequences (Menon and Goodyear, 1999), however the property of spin-echo to refocus extravascular dephasing and hence reduce 'downstream' BOLD contributions for better specificity has led to a series of papers advocating spin-echo fMRI at UHF (Yacoub et al., 2005); some researchers therefore also today advocate $T_{2}$-contrast for submillimeter BOLD imaging (De Martino et al., 2013b; Kemper et al., 2014). The main challenges associated with spin-echo fMRI are the much increased RF power deposition which puts tight limitations on the achievable acquisition rate and the imaging volume that can be covered, as well as $\mathrm{B}_{1}{ }^{+}$inhomogeneity which causes large spatial nonuniformity in the signal excitation of spin-echo imaging. Gradient-echo imaging with either 2D slice-selective EPI or 3D volume-selective EPI readout is therefore the choice in most cases, and if acquired at sufficient spatial resolution the undesired signal contributions from the larger draining veins or pial vasculature can be identified directly and removed from subsequent analysis (Curtis et al., 2014; Menon, 2002). An alternative to spin-echo EPI that does not require a SAR intensive 180 degree refocusing pulse is the SSFP (steady state free precession) sequence (Barth et al., 2010; Goa et al., 2014; Miller et al., 2006; Poser et al., 2010; Scheffler et al., 2001; Scheffler and Ehses, 2016) which also provides a spin-echo like $T_{2}$ contrast. SSFP sequences come at lower temporal resolution than typical EPI sequences since single-shot acquisition cannot be realized due to the need to keep TR short and acquire the signal as closely as possible to the subsequent RF pulse.

Many BOLD applications at UHF translate the CNR gain into acquisitions at improved spatial and/or temporal resolution in order to obtain new insights into the functional organization of the brain. Smaller voxel sizes allow us to functionally resolve the finer structures in the cortical or subcortical architecture that are not easily seen at clinical field strengths. This has been applied to the measurement of cortical layer-specific BOLD activations (Koopmans et al., 2010; Olman et al., 2012; Polimeni et al., 2010; Siero et al., 2011; Trampel et al., 2012) and even mapping of intra-cortical connections or probing the columnar organization of cortex (Yacoub et al., 2008). In subcortical areas small voxels can be used for the functional segregation of nuclear sub-regions (De Martino et al., 2013a; Satpute et al., 2013). Thanks to the now widespread availability of UHF scanners, such advanced applications of BOLD fMRI have already evolved to a level of maturity that they no longer remain accessible to only few privileged researchers. Considering the rapid advance of acquisition, reconstruction and analysis techniques we have experienced in only the past few years, we can reasonably expect that the persistent effort in further advancing acquisitions technology will continue to push the boundaries of neuroscience applications in the coming years, and that these developments will increasingly be driven by applications and neuroscientific research questions. Improvements in temporal fMRI resolution bring other advantages by more tightly sampling the hemodynamic signals. Despite the sluggishness of the BOLD response, shorter TR acquisition by itself has been demonstrated to increase statistical power for example in resting state fMRI where it allows for improved functional parcellation of brain regions and even facilitates mapping the shortterm dynamic reorganization of resting state network topography (Tian et al., 2013). High frame rates in task fMRI not only allow for tighter sampling of the BOLD response but also help better resolving the physiological noise contributions originating from respiration, heartbeat as well as local pulsation and gross motion, thereby easing physiological noise reduction in subsequent post-processing (Glover et al., 2000; Griffanti et al., 2014). Together this for instance enables the measurement of hemodynamic delays within and across brain regions, and allows inferences to be made on the hierarchical organization and directionality of brain signals (Lee et al., 2013). Moreover, a recent study has detected oscillatory fMRI signals in response to stimuli oscillating at up to $0.75 \mathrm{~Hz}$ within single scan sessions (Lewis et al., 2016a), and these responses, although small, were an order of magnitude larger than predicted by canonical linear models. In the same study it was shown through simulation based on a biophysical model of the hemodynamic response that the BOLD response should become faster for rapidly varying stimuli, enabling the detection of higher frequencies than expected (Lewis et al., 2016b). It is therefore expected that the high SNR and the high spatial-temporal resolution fMRI afforded at UHF will likely be the key for further investigation in this area, with exciting potential in mapping neural oscillations directly throughout the brain. Furthermore, the investigation of the mechanisms of neurovascular coupling that ultimately leads to the largely phenomenological BOLD response remain an important research topic that spans different modalities (O'Herron et al., 2016). Recent reports in the optical imaging literature indicate that there is highly localized regulation of capillary blood flow (Devor et al., 2012), implying a direct link between the neuronal activation and hemodynamic fMRI signal that could be achieved at microscopic voxel resolution. Thus, the limiting factor that compromises the spatial specificity of fMRI is the spatiotemporal resolution we can achieve with current fMRI acquisitions. While true microscopic resolutions with (f)MRI will likely never be achieved, it is clear that UHF and advanced acquisitions and reconstruction are the key ingredients towards mastering this challenge. Fig. 1 shows examples of BOLD EPI applications that have been enabled by the advances in SMS acquisition with controlled aliasing, illustrating the paradigm shift that the field of fMRI has undergone in the past 5 years. Fig. 2 shows comparisons of rs-fMRI data acquired using the Human Connectome Project (HCP) protocols at $3 \mathrm{~T}$ and $7 \mathrm{~T}$ at $2 \mathrm{~mm}$ and $1.6 \mathrm{~mm}$ isotropic resolution respectively (figure and results adapted from Vu et al. (2016)). Here, the increase in SNR and CNR of UHF are shown to improve rs-fMRI results, while the ability and desire to acquire data at higher spatial resolution is shown to negate some of this benefit, particularly when higher in-plane acceleration is employed to counteract the increased distortion and blurring.

\section{Vascular space occupancy (VASO) fMRI}

Since the BOLD signal contains a blood volume contribution that is well localized to the capillaries, efforts have been made to measure 


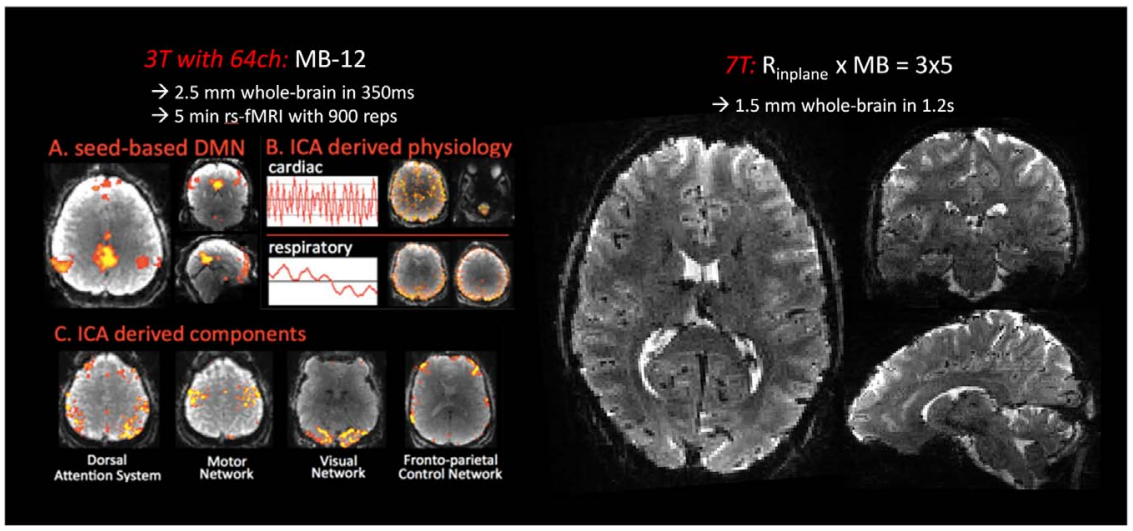

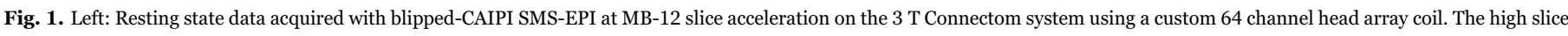

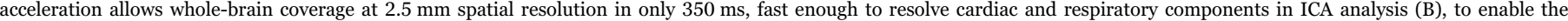

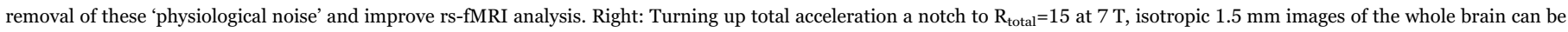

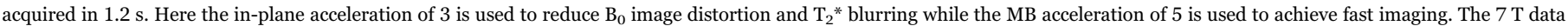
were acquired on a 32-channel head array built in-house at MGH on a scanner with $\mathrm{SC} 72$ whole-body gradients $(70 \mathrm{mT} / \mathrm{m}, 200 \mathrm{mT} / \mathrm{m} / \mathrm{ms})$.

vascular volume changes directly (Lu et al., 2013, 2003) Also early work on hemodynamic modelling to understand the temporal relationship between the observed BOLD signal, cerebral blood flow (CBF), cerebral blood volume (CBV) and the cerebral mean rate of oxygen consumption $\left(\mathrm{CMRO}_{2}\right)$ attempted to measure the $\mathrm{CBV}$ contribution in isolation (Buxton et al., 1998; Mandeville et al., 1999). However until a decade ago, this was only possible using superparamagnetic MION (monocrystalline iron oxide nanocolloid) contrast agents and hence limited to animal studies, which, together with the fact that such studies required anesthesia led to some discussion on the translatability of the resulting models to the hemodynamics in the human (Kim et al., 2013). Lu et al. (2003) proposed the so-called vascular space occupancy (VASO) method, which in its basic form is a single-shot inversion recovery sequence that acquires a single image at the nulling point of intravascular blood. To achieve this, the $T_{1}$ of blood must be known precisely to allow separation from the surrounding gray matter. Since the vascular volume increases during activation and this contribution to the voxel signal is removed by the inversion, a negative signal change is observed during task activation. Animal experiments at 9.4 T with layer specific fMRI could confirm good agreement between the specificity of VASO and MION contrast (Jin and Kim, 2006), and even demonstrated improved specificity of slab-selective VASO over the SE BOLD signal (Jin and Kim, 2008). The VASO method provoked some interest for human use, and at $3 \mathrm{~T}$ was developed further to provide increased brain coverage using the MAGIC (Lu et al., 2004) scheme or single-shot 3D GRASE (Donahue et al., 2009; Poser and Norris, 2009). While this allowed application to whole-brain cognitive fMRI to be demonstrated (Poser and Norris, 2011), VASO for some time remained a niche technique for mechanistic investigations and limited to low spatial resolutions. A review on the development of VASO can be found in Lu and van Zijl (2012) and Lu et al. (2013).

Considering the lengthening of $\mathrm{T}_{1}$ with field strength (requiring longer TR) and the convergence of $\mathrm{T}_{1}$ of blood and gray matter (making them harder to separate), the implementation at UHF is not entirely straightforward (Hua et al., 2013). The prospect however of translating the higher SNR at UHF into high spatial resolution in order to capitalize on the superior specificity of VASO evidenced in the animal literature, provided compelling reason to pursue VASO at high field (Hua et al., 2013). Considerable effort has since gone into the adaptation of VASO for UHF human fMRI, and slab-selective VASO with BOLD compensation at $7 \mathrm{~T}$ has now been applied to study neurovascular coupling, layer-dependent fMRI (Huber et al., 2014), and layer-resolved resting state fMRI at sub-millimeter resolutions (Huber et al., 2016a); in each case VASO exhibited better specificity than the concurrently measured BOLD signals. The success of broader application of VASO critically hinges on the advances in parallel image reconstruction and SMS technology to provide a powerful means to address the limited volume coverage: recent work showed SMS-VASO for blood volume fMRI with near whole-brain coverage, high-resolution mapping of spatially distinct brain areas, and sub-millimeter fMRI with multi-slice coverage (Huber et al., 2016). For submillimeter resolutions, VASO with 3D-EPI readout has since been found to provide yet superior SNR at high spatial resolution as compared to 2D-SMS readout; for larger voxel sizes the reverse is the case, as can be seen in Fig. 3. 3D-EPI based VASO also showed significantly reduced sensitivity to motion than SMS-VASO in breathhold experiments (Huber et al., 2016b). Despite VASO's specificity advantages over BOLD at UHF, its remaining limitations with regards to spatial coverage and temporal resolutions will likely prevent its acceptance for wide-spread use for 'standard fMRI': While BOLD fMRI with SMSEPI at $1.5 \mathrm{~mm}$ resolution can readily be achieved with TR of $1.5 \mathrm{~s}$ or less, TR in VASO is dictated by the need for magnetization recovery and typically 2.5 to $3 \mathrm{~s}$, irrespective of volume coverage. It may nevertheless be expected to continue its rapid advance for special applications like layer connectivity within brain regions and studies into the laminar and columnar architecture of human cortex.

\section{Perfusion imaging using ASL}

Perfusion imaging using MRI is typically performed non-invasively using ASL which has been developed since the late 1980s (Detre et al., 1992; Williams et al., 1992). ASL allows measuring regional cerebral blood flow in health and disease, although in a clinical setting dynamic susceptibility contrast (DSC) is often preferred for the higher sensitivity. For a recent review on ASL, the reader is referred to (Koretsky, 2012). ASL at higher resolutions and hence reduced voxel volume lead to proportional reduction in the SNR of ASL acquisitions, which render high-resolution perfusion imaging particularly challenging. In practice, to obtain sufficient SNR in ASL at $3 \mathrm{~T}$, low spatial resolutions (above $3 \mathrm{~mm}$ isotropic) are utilized. Therefore, only a few studies have shown CBF maps with in-plane resolution below $1.5 \mathrm{~mm}$ (Pfeuffer et al., 2002b; Zuo et al., 2013). It is worth noting that these studies utilized diverse acquisition approaches - SE EPI, GE EPI and turbo-FLASH (TFL), but were all performed at $7 \mathrm{~T}$, indicating its potential for highresolution CBF imaging. Performing ASL at UHF promises to be advantageous because of increased image SNR and longitudinal relaxation times (Gardener et al., 2009; Ivanov et al., 2016b). For instance, $7 \mathrm{~T}$ ASL provides improved white matter perfusion measurements (Gardener and Jezzard, 2015). Despite the aforementioned gains, UHF ASL has not found widespread use in humans due to 

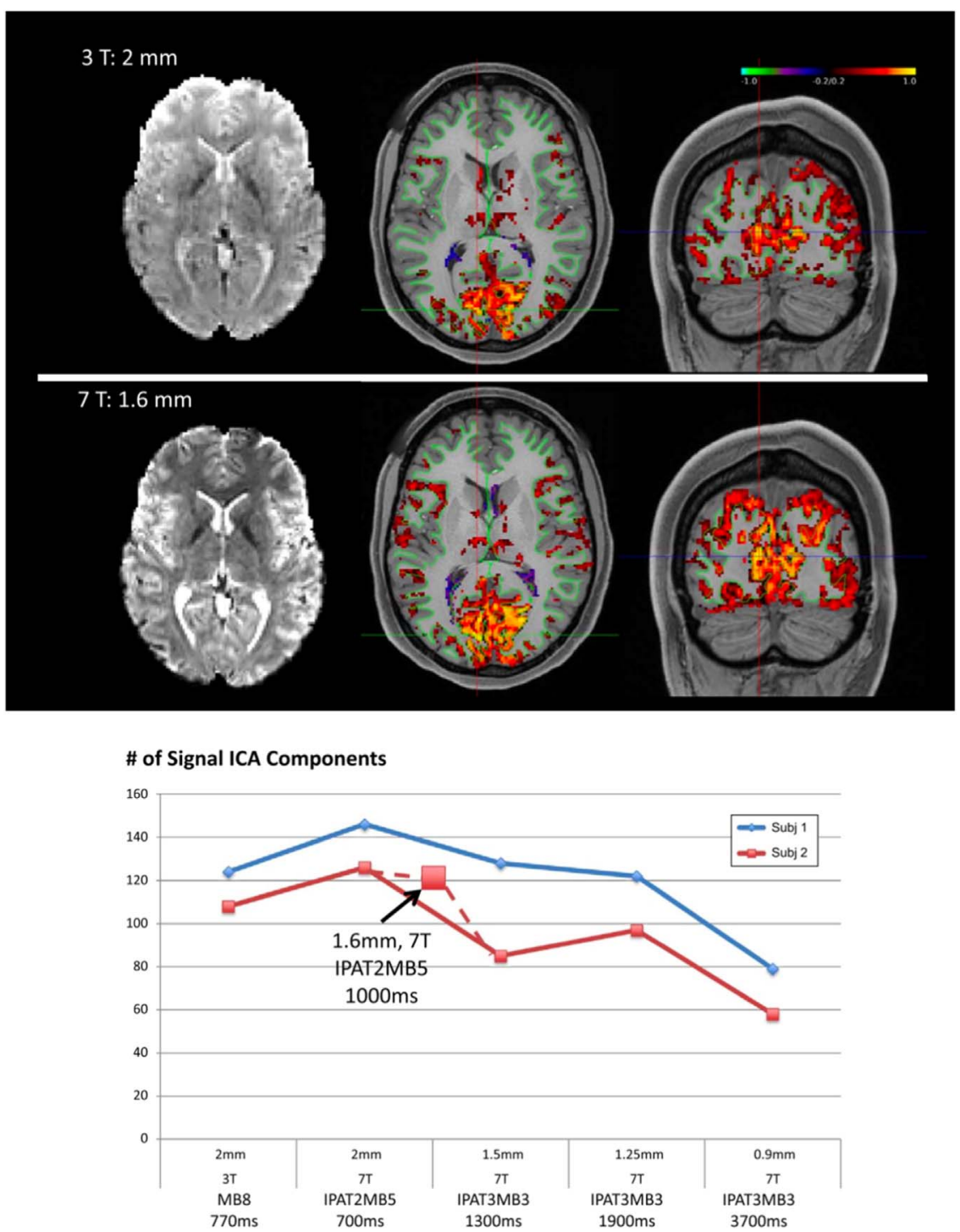

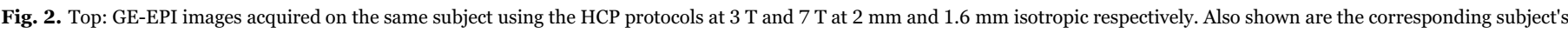

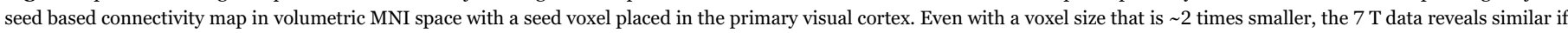

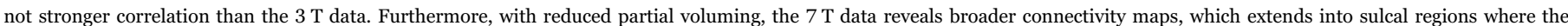

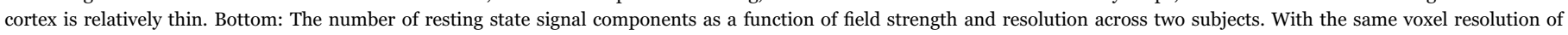

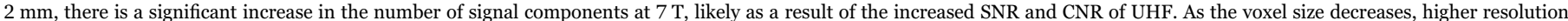

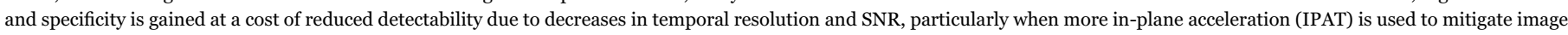
distortion and blurring. Figure adapted from Vu et al. (2016).

significant technical challenges. First, the spatial homogeneity and efficiency of the labeling are significantly degraded at UHF due to $\mathrm{B}_{0^{-}}$ and $\mathrm{B}_{1}{ }^{+}$-inhomogeneities (Luh et al., 2013; Teeuwisse et al., 2010). Second, SAR constraints at UHF constrain the utilization of some widely-used $3 \mathrm{~T}$ ASL techniques, such as pCASL labeling background suppression and spin-echo-based readouts. As a consequence, to remain within SAR limits, many UHF ASL implementations employ poor temporal resolutions (Bause et al., 2016; Ghariq et al., 2012; Luh et al., 2013; Zuo et al., 2013). Recently, Ivanov and colleagues demonstrated a pulsed ASL (FAIR) approach at $7 \mathrm{~T}$ utilizing optimized tr-FOCI inversion pulses, dielectric pads and SMS EPI readout capable of whole brain coverage with moderate spatial resolution and TR similar to $3 \mathrm{~T}$ (Ivanov et al., 2016b). The same labelling approach has been combined with a 3D-EPI readout to achieve higher SNR CBF imaging at sub-millimeter spatial resolution (Ivanov et al., 2016a), in line with the findings from VASO where 3D-EPI was found to outperform 2D-EPI in case of very small voxel size (Huber et al., 2016c). Moreover, these initial sub-millimeter ASL data are suggesting a superior specificity to BOLD (see paper by Huber et al. in the Special Issue 'Prospects for cortical laminar MRI').

\section{Diffusion imaging}

Diffusion MRI (dMRI) is an inherently low-SNR imaging technique. The increase in SNR afforded by UHF could potentially be used to increase the spatial (k-space) and angular diffusion (q-space) resolution of dMRI, to improve its utility as a tool in studying fine-scale structures and complex diffusion properties. Nonetheless, unlike fMRI, the $\mathrm{T}_{2}$ shortening at UHF is detrimental to dMRI as it significantly decreases the signal of the typically long TE spin-echo dMRI acquisition (long TE due to diffusion gradient encoding). To illustrate, for a typical dMRI acquisition with TE of $65 \mathrm{~ms}$, the SNR reduction in whitematter due to a $\mathrm{T}_{2}$ shortening from $77 \mathrm{~ms}$ at $3 \mathrm{~T}$ to $50 \mathrm{~ms}$ at $7 \mathrm{~T}$ (Cox and Gowland, 2008) is a factor of $1.6 \times$, which wipes out a large portion of the $2.3 \times$ gain in SNR from moving to $7 \mathrm{~T}$. This $\mathrm{T}_{2}$ shortening coupled with the issue of $\mathrm{B}_{1}{ }^{+}$inhomogeneity and SAR, make squeezing out good 
temporal signal to noise in 3D-EPI VASO and 2D-SMS VASO across voxels sizes

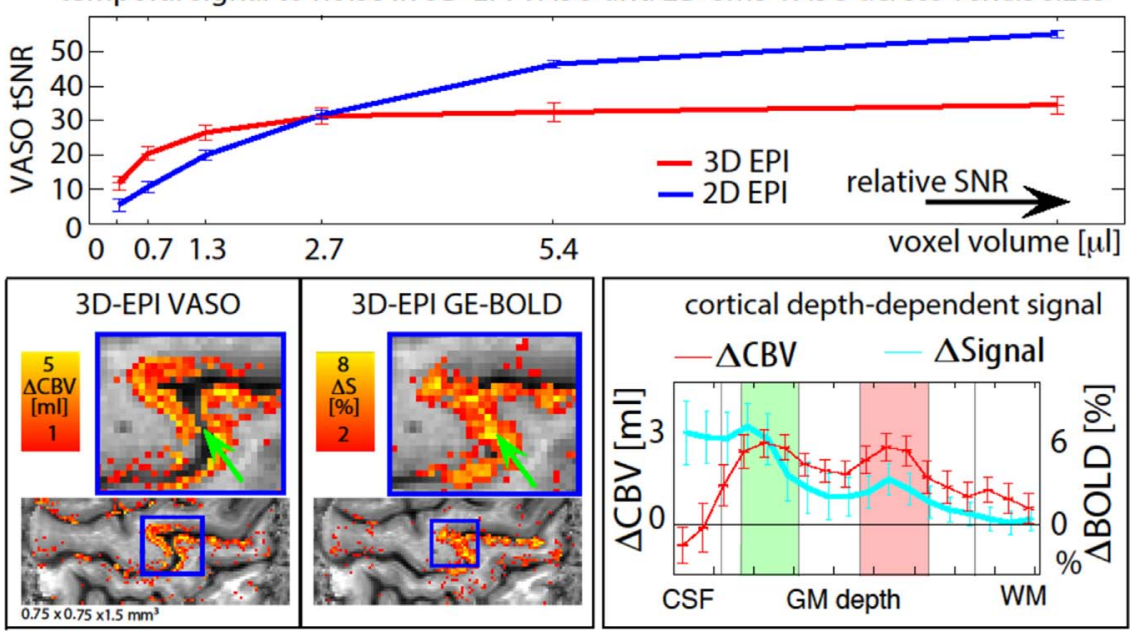

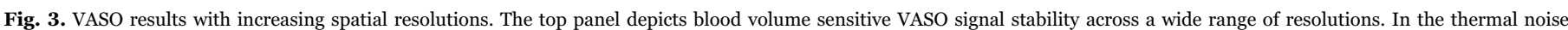

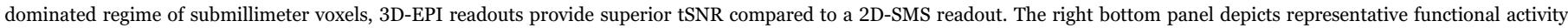

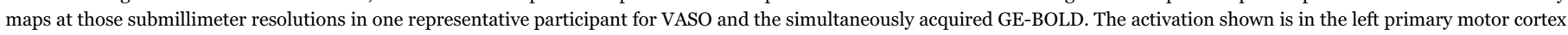

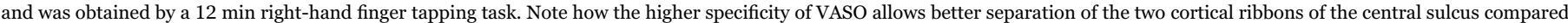

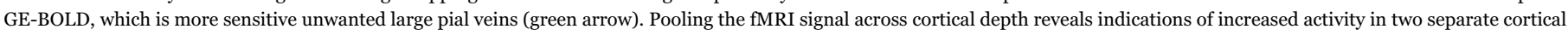
layers (highlighted red and green areas in bottom right panel). For more information about the acquisition parameters of the depicted data, please see Huber et al. (2016b).

performance from UHF dMRI an uphill battle. Nonetheless, high quality diffusion imaging at high spatial resolution has been achieved, largely through keeping to short TE acquisitions by targeting relatively low b-value encoding and by employing large in-plane acceleration/ multi-shot and/or zoom imaging approaches to limit the EPI encoding burden (Eichner et al., 2014a; Heidemann et al., 2012, 2010; von Morze et al., 2010). Moreover, recent persistent efforts in dMRI at $7 \mathrm{~T}$ with SMS-EPI (Vu et al., 2014) and multi-slab 3D-EPI (Wu et al. 2016a, 2016b) have also shown promising results with high spatial resolutions.

\section{Susceptibility imaging}

Structural imaging with susceptibility contrast through phase imaging, susceptibility-weighted imaging (SWI) and quantitative susceptibility mapping (QSM) have all been shown to gain dramatic improvement in $\mathrm{CNR}$ at $\mathrm{UHF}$, yielding exquisite intracortical and subcortical contrast (de Rochefort et al., 2010; Deistung et al., 2008; Duyn et al., 2007; Fukunaga et al., 2010; Haacke et al., 2004; Shmueli and Zwart, 2009). With QSM, the orientation bias in the susceptibility signal can be further removed to provide structurally accurate quantitative tissue information at a cost of some spatial blurring (Bilgic et al., 2012; de Rochefort et al., 2010; Liu et al., 2011; Marques and Bowtell, 2005). Alternatively, repeated and hence time-consuming measurements can be performed in which data are acquired at multiple head orientations (Liu et al., 2009). The high CNR and SNR of susceptibility contrast acquisitions at UHF have enabled us to strive for very high isotropic submillimeter resolutions in these imaging applications. Here, the developments of 3D-EPI with CAIPI and Wave-CAIPI GRE acquisitions (outlined below) have provided the much-needed improvement in acquisition speed to allow such high-resolution data to be acquired in vivo within reasonable measurement time. Example QSM data from these two sequences is shown in Fig. 4.

\section{$T_{1}$-weighted and $T_{2}$-weighted imaging}

$\mathrm{T}_{1} \mathrm{w}$ and $\mathrm{T}_{2} \mathrm{w}$ structural imaging also benefits from higher resolutions afforded through higher SNR at UHF. The increased resolution improves the ability for these imaging techniques to serve as tools for neuroscience and clinical research. In particular, with its excellent gray matter/white matter contrast, $\mathrm{T}_{1} \mathrm{w}$ MPRAGE (Mugler et al., 1992;
Mugler and Brookeman, 1990) has been widely used for (automatic) brain segmentation (Dale et al., 1999; Fischl et al., 1999), where segmentation precision has improved with the increased resolution. This has allowed us to better study healthy brain development, measure cortical thickness/volume (Fischl and Dale, 2000), and investigate into pathological conditions such as those associated with neurodegeneration. On the other hand, it is important to note that the $\mathrm{T}_{1}$ lengthening and $\mathrm{T}_{2}$ shortening at UHF can have some negative impact on SNR and also lengthen contrast encoding period/dead-time of these acquisitions. The imaging parameters should therefore be optimized to account for these $T_{1}$ and $T_{2}$ changes to ensure high quality imaging with optimal contrast profile.

The increase in $\mathrm{B}_{1}{ }^{+}$and $\mathrm{B}_{0}$ inhomogeneity at UHF also affects the imaging quality of these acquisitions. Sequence modifications particularly for MPRAGE, have been proposed to overcome these issues. In particular, MEMPRAGE (van der Kouwe et al., 2008), which utilized multi-echo acquisition at high bandwidth, has been proposed to reduce $\mathrm{B}_{0}$ inhomogeneity related image distortion. Moreover, MP2RAGE (Marques et al., 2010) which acquires data at two different inversion times, has also been proposed to create a $\mathrm{T} 1 \mathrm{w}$ image free of proton density contrast, $\mathrm{T}_{2}{ }^{*}$ contrast, reception bias field, and, to first order $\mathrm{B}_{1}{ }^{+}$inhomogeneity. Increased coil sensitivity variation at UHF (Ohliger et al., 2003; Wiesinger et al., 2004) on the other hand presents the opportunity for higher accelerations and faster imaging, but the speed gain here could be limited, particularly in SMS imaging where higher SAR is expected at higher slice accelerations. Fig. 5 shows a SMS $\mathrm{T}_{2}$-TSE acquisition at a high slice acceleration factor (Norris et al., 2014) where the use of PINS refocusing pulses (Norris et al., 2011) has helped alleviate this issue.

\section{Time-of-flight magnetic resonance angiography (TOF-MRA)}

TOF-MRA acquisition achieves high vessel contrast by utilizing short TRs to keep the stationary tissue signal suppressed relative to the moving blood signal in the vessel. The $\mathrm{T}_{1}$ lengthening effect of UHF helps improve the suppression of the stationary tissue signal to improve CNR (von Morze et al., 2007; Zwanenburg et al., 2008). This coupled with the increased SNR of UHF provides for the ability to achieve very high spatial resolution TOF-MRA. Fig. 6 shows a highly detailed TOF-MRA (no contrast injection) data at $7 \mathrm{~T}$ where vascular branches down to the 3rd and 4th order are observed.

The increase in resolution naturally comes at a cost of increased 


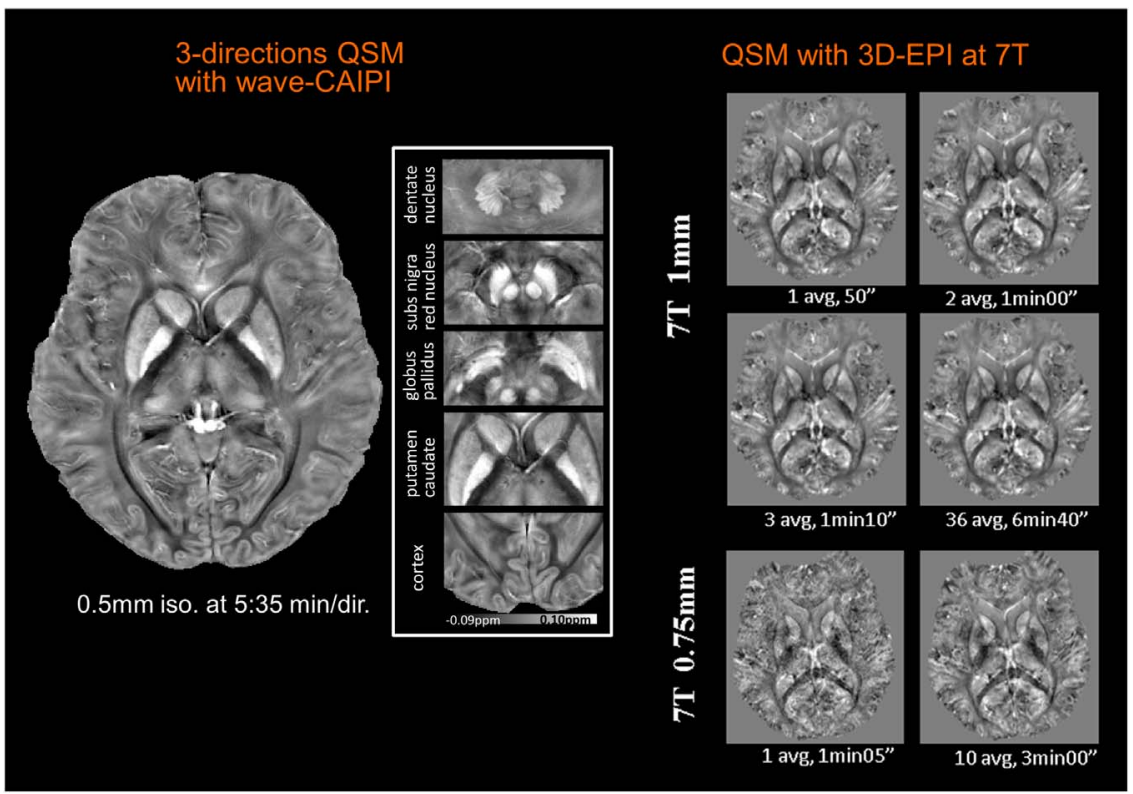

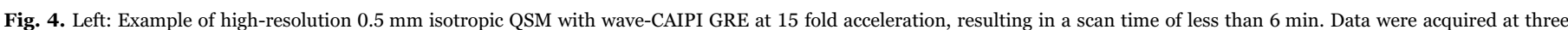

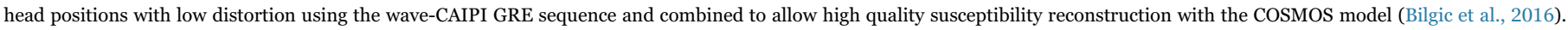

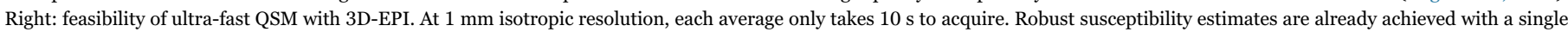

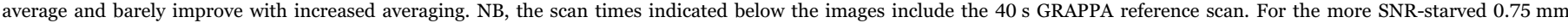

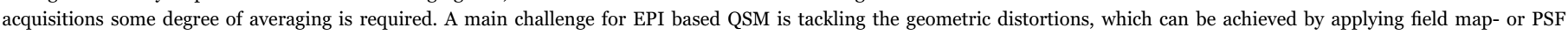
unwarping to the complex source image. The 3D-EPI data are taken from Poser et al. (2015).

encoding burden, where longer scan time leads to more motion sensitivity. A recently proposed Multiband-MultiSlab approach (MBMS) for TOF-MRA (Schulz et al., 2016) can help mitigate this issue by allowing for faster imaging as well as an increase in CNR. With MB-MS, multiple thin slabs are acquired simultaneously (with CAIPI shift between the slabs) to allow for good slab-acceleration and faster imaging. Furthermore, the MB-MS approach also increases CNR through the use of thinner slab acquisition (relative to standard slab acquisition) with reduced blood saturation effect and the increase in magnetization transfer contrast from the use of multi-slab pulses.

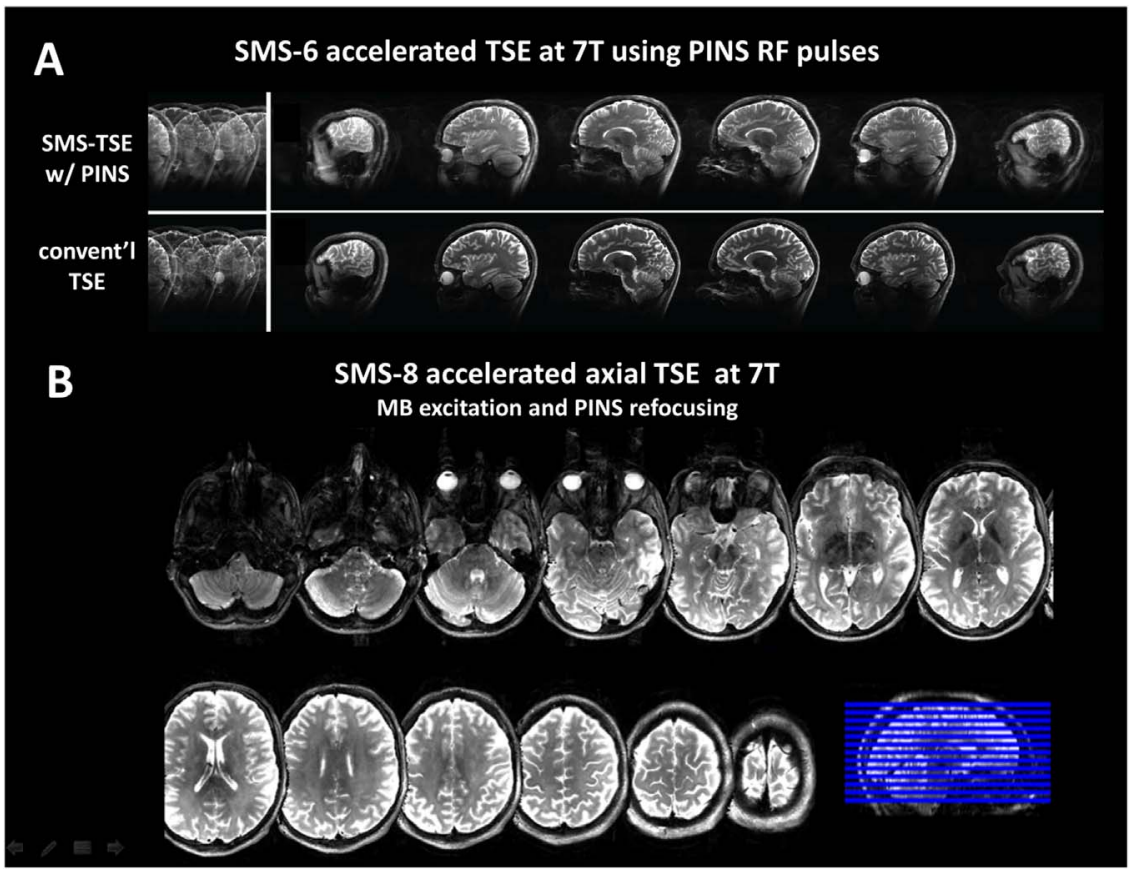

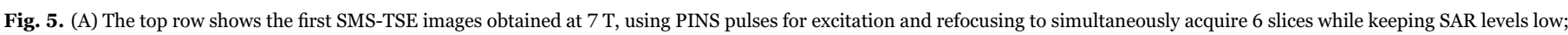

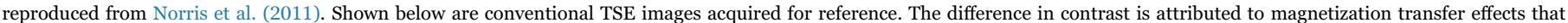

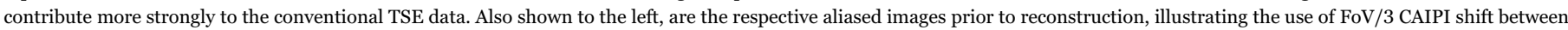

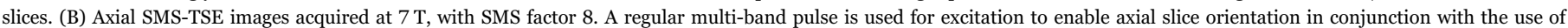

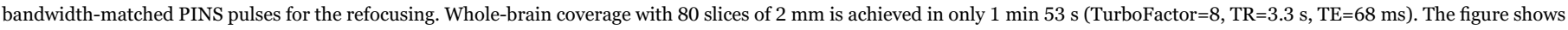

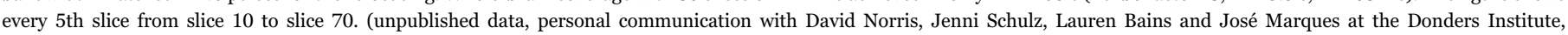
Nijmegen, NL). 


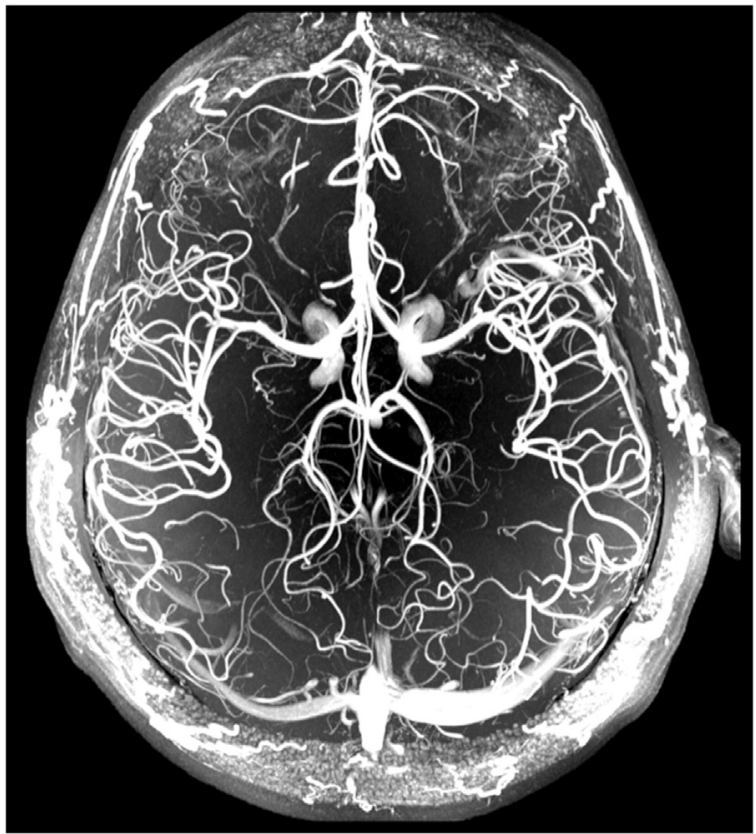

Fig. 6. High SNR and CNR TOF-MRA data at $7 \mathrm{~T}$. Partial MIP (maximum intensity projection) of $300 \mu \mathrm{m}$ isotropic whole-brain TOF-MRA is shown, where detailed vasculatures can be observed without a need for contrast injection (unpublished data, personal communication with Jonathan Polimeni, Athinoula A. Martinos center, Massachusetts General Hospital, USA).

\section{Meeting the need for speed}

The main conundrum with high-resolution acquisitions that are usually desired at UHF is the correspondingly increased measurement time that is required. This can make in vivo applications challenging or outright impracticable. In fact, modern fMRI applications such as those mentioned above generally rely on high spatial and temporal resolution at the same time, and recently popular anatomical protocols for quantitative MRI parameter mapping now include high-resolution scans of different image contrast which increases the total scan time. The problem is amplified in studies involving patients, elderly and children, or other populations that have difficulty remaining still over an extended period of time. Moreover, subject motion frequently renders images worthless and adequate real-time correction techniques that are routinely applicable are largely lacking. With the recent release of the first FDA approved commercial $7 \mathrm{~T}$ system and the foreseeable use of rather expensive UHF imaging for diagnostic use, one may add economic considerations to what can be summarized as a pressing need for speed. This poses significant requirements on the acquisition hardware (high field, many-coil transmit and receiver arrays, stronger gradients, better $\mathrm{B}_{0}$ shimming), MR physics (smart sequence sampling schemes and reconstructions, coil compressions) as well as powerful soft- and hardware solutions to achieve near real-time image reconstructions (many-count CPU and GPU systems). In particular, the exploitation of UHF MRI now relies critically on parallel imaging (PI) technology, which has gone through an extraordinary evolution over the past decade. SENSE (Pruessmann et al., 1999) or GRAPPA (Griswold et al., 2002) type reconstructions enable an acquisition speedup by simultaneously receiving the MR signal with multiple receiver coils and whose distinct receive sensitivity profiles provide spatial encoding power that can replace sampling steps in the imaging sequence. Head and body coil arrays with 32 or more coil elements have become a standard at clinical field strengths, yet only rarely do routine protocols apply acceleration factors $R$ of more than 3 to 4 even in volumetric acquisitions. This is because conventional parallelaccelerated acquisitions incur a $\operatorname{sqr}(R)$ SNR penalty as fewer samples are acquired, and in addition a so-called g-factor noise penalty that depends on the array's channel count and geometry in relation to the acceleration factor and acceleration direction (Pruessmann et al., 1999). As a result, traditional PI sampling schemes in 2D slice imaging (e.g. for $\mathrm{R}=3$ simply acquire only every $3 \mathrm{rd} \mathrm{k}$-space line which effectively reduces the FoV by factor 3 ) have remained limited to modest acceleration factors, even with highly advanced receive coil technology. Fortunately at UHF, the sensitivity profiles of the individual coil elements become more distinct which results in an increased encoding power and hence reduced g-noise penalty (Ohliger et al., 2003; Wiesinger et al., 2006, 2005). This advantage can be translated into higher acceleration factors, bringing $R=5$ into the range of what is reasonably achievable along a single direction. Nevertheless the fundamental limitations of $1 \mathrm{D}$ acceleration remain as they do not make full use of the coil array's encoding capability: which is typically spread across all spatial directions (i.e. 3D) in common soccer-ball designs (Wiggins et al., 2009, 2006).

The most recent advances in the domain of parallel imaging, simultaneous multi-slice acquisition and controlled aliasing (CAIPIRINHA, in the following referred to as CAIPI), have revolutionized both 2D slice (Breuer et al., 2005) and 3D volumetric sampling (Breuer et al., 2006). In essence, the idea of SMS sampling was not new and reaches far back to the days before parallel imaging even came along. Of particular note is a technique known as POMP (Glover, 1991) that already incorporated the main features that now enable modern day SMS sequences: multiband excitation and phase encoding along the multiple simultaneous slices. Shortly after the first demonstrations of parallel imaging for 2D acquisitions, David Larkman then was the first to demonstrate truly slice-accelerated acquisitions, ie. simultaneous excitation of multiple slices at once by means multi-band RF pulse, and subsequent SENSE reconstruction to separate the slices using coil sensitivity information (Larkman et al., 2001). Most importantly, the simultaneous acquisition of the signals originating from multiple simultaneous slices took the same time as would normally be spent on a single slice. A ground-breaking development at the time, the publication remained largely overlooked for several years until Breuer and colleagues gave the concept a significant methodological push forward through CAIPIRINHA, which enabled higher acceleration via better use of coil sensitivity information across multiple dimensions. Even then the community did not yet seem to fully embrace the great potential of the controlled aliasing principle, and SMS was not worked on by many. A main reason for this may be the lack of widespread availability of multi-channel receive coils with a total channel count greater than eight, or coils with elements along the head-foot direction which limits acceleration capability. As one of the pioneers who recognized early on that EPI would be 'the' major beneficiary of SMS acquisition, Nunes and colleagues presented an implementation of SMS-EPI with CAIPI slice shift (Nunes et al., 2006) shortly after the first CAIPIRINHA paper; but since this first implementation left a significant blurring artifact in the reconstructed images the work did not at the time attract the attention that it now receives in retrospect. The turning point, at least for the fMRI community, came in the year 2010 which then saw two highly promising demonstrations for EPI at once: 3D-EPI which for the first time allowed nominal acceleration time savings by slice/through-plane undersampling of the secondary phase-encoding direction plus any partial Fourier (Poser et al., 2010), and at the same time Moeller et al. presented a first version of SMS-EPI for fMRI showing faster sampling at UHF (Moeller et al., 2010). Both were still without controlled aliasing. It was however not until the first convincing demonstration of the actual benefits that fast sampling in fMRI can bring about (Feinberg et al., 2010) and the development of the blipped-CAIPI method (Setsompop et al., 2012) that allows artifact free controlled aliasing in EPI, that the advancement of SMSCAIPIRINHA and related reconstruction methods began to be pushed forward in a well-orchestrated manner and at a rate the MR methods community has rarely witnessed before. CAIPI now comfortably allows PI accelerations up to a factor of about 12 with acceptable noise penalty 
and has enabled applications that were unthinkable only a few years ago. The key to CAIPI is to acquire an imaging signal that originates from a 3D distribution in space, this can be a contiguous imaging slab or multiple simultaneously excited slices in different locations. The undersampling alias can then be distributed more evenly over more than one spatial direction (hence "controlled aliasing"), which increases the spatial distances between overlapping signals in the reduced field of view (FoV) and thereby exhibits lower g-factor penalty (Breuer et al., 2006). This advantage again translates into higher acceleration factors becoming possible. Intriguingly, the CAIPI technique also allows coil sensitivity information along one dimension to be invested into accelerating along an orthogonal dimension in which the coil array may provide little or no spatial encoding (Breuer et al., 2005; Setsompop et al., 2012), and so with CAIPI, it is also possible to perform slice acceleration in SMS-EPI using a coil array with no coil sensitivity variation along $\mathrm{z}$ (where coils are physically distributed solely along $\mathrm{x}, \mathrm{y}$ in a single $\mathrm{z}$ row) by leveraging the coil sensitivity information along the phase encoding direction. This is what constitutes the main "secret" that, for instance, enables SMS-EPI with factor 12 acceleration along a single direction, the slice direction (Setsompop et al., 2016; Xu et al., 2013). Here coil sensitivity along both the phase encoding and the slice directions are contributing to achieve such a high acceleration. Such high accelerations are also now being used routinely with a 32-channel coil at $7 \mathrm{~T}$, although here the acceleration is typically used in a distributed fashion along both the slice and the phase encoding directions $\left(M B \times R_{\text {in-plane }}\right)$ to mitigate the increased image distortion and blurring associated with higher field and the typically higher targeted spatial resolution (Uğurbil et al., 2013; Vu et al., 2016).

From a pure sampling perspective, 2D-SMS and 3D volumetric CAIPI are equivalent: they can be represented in the same 3D k-space picture (Zahneisen et al., 2014) and if so desired even reconstructed in exactly the same manner using SENSE (Zahneisen et al., 2015). The $\mathrm{N}_{\text {sms-factor }}$ slices in a SMS slice group can then be viewed as being centered in voxels of voxel size equal to the slice separation. Note moreover, that from this sampling perspective, a CAIPI acquisition is no longer appropriately described by the undersampling factors in one and the other direction, but characterized by the total amount of undersampling and a sampling pattern, that in turn gives rise to an aliasing pattern (Breuer et al., 2006).

In practical sequence implementation, the staggered sampling in $\mathrm{k}-$ space can be achieved in two ways. The now historic literature on simultaneous acquisitions using shifted 2D slices, even before the advent of parallel imaging, such as the POMP method (Glover, 1991) used sets of different multi-band pulses in which, between adjacent kspace lines, the phase of the slices to be shifted was cycled so as to effect a slice-shift according to the Fourier shift theorem. The same approach was used in the original demonstration of slice CAIPIRINHA (Breuer et al., 2005). It lends itself well to SMS with a small number of slices, but becomes more impractical for high SMS factors as the number of distinct RF waveforms is equal to the number of simultaneous slices. More importantly, slice shift by RF phase only works in slice-selective sequences with "one line per excitation" or segmented EPI or TSE echo trains, but it does not work in single-shot echo-train sequences like EPI, or any volumetric 3D sequences. Recognizing however that the distinct multi-band pulses effectively perform a phase-encoding of the 'voxels' defined by the simultaneously excited slices, the encoding can be replaced by gradient moments, which unlike RF can also be applied during echo-train readouts. This enabled demonstration of volumetric CAIPIRINHA (Breuer et al., 2006) and ultimately blipped-CAIPI EPI (Setsompop et al., 2012) that is now the standard way in which to perform SMS-EPI. Fig. 7 shows examples of the gradient schemes in blipped-CAIPI EPI, and explains by reference to the k-space representation why the identical EPI readouts and reconstruction can be used in SMS-EPI as well as 3D-CAIPI-EPI encoding (Narsude et al., 2016; Zahneisen et al., 2015).
With regards to reconstruction, SMS-EPI can be reconstructed using variants of any of the common Cartesian SENSE (Larkman et al., 2001; Zahneisen et al., 2015), GRAPPA (Setsompop et al., 2012), or SENSE/GRAPPA hybrid approaches (Blaimer et al., 2006; Koopmans, 2016; Moeller et al., 2010). A small overview of the different options can be found in the review by Barth et al. (2015). Today however, the by far most commonly used SMS reconstruction is slice-GRAPPA (Setsompop et al., 2012), which uses a multi-kernel approach to fit a separate GRAPPA kernel for each slice to be unfolded. This has the inherent advantage of being more robust to motion contamination, and, unlike the other reconstruction approaches, readily allows for the incorporation of a penalty term to suppress slice-leakage, i.e. residual aliasing/ghosting between slices (Cauley et al., 2013).

In practice, it depends strongly on the type of sequence to what extent the undersampling factor translates literally, into a temporal acceleration factor. In a volumetric 3D gradient echo sequence that samples one line of $k$-space per TR, the speedup factor is equal to the total undersampling no matter what the sampling pattern. In a 2D EPI sequence, in-plane undersampling shortens the EPI readout, which reduces geometric distortions and/or enables a larger matrix size to be acquired at a given echo time; the undersampling does not however result in appreciable temporal acceleration, as the TR remains largely dependent on the TE required for the BOLD signal to develop, or the large duration taken up by the diffusion preparation in the case of dMRI. As such, standard PI has been of little value in reducing the TR of 2D EPI. This is fundamentally different in 3D and SMS echo-planar acquisitions where the undersampling can be applied along an orthogonal phase-encoding direction, allowing entire sampling blocks (excitation, any preparation and EPI readout) to be skipped and which results in temporal acceleration equal to the nominal undersampling factor along that second phase encoding dimension. This has huge implications for the achievable TR reductions and has been exploited in both volumetric 3D-EPI and more recently in SMS-EPI. The ability to speed up 2D single-shot EPI by an order of magnitude or more, has within only a couple of years resulted in the technique becoming a widely accepted new standard, and this includes its application in large populations studies such as the Human Connectome Project or ABCD Study in the US, or the UK Biobank and Rhineland studies in Europe.

Next to the self-evident advantage of shorter TR, there are two important characteristics that make 2D SMS-EPI the powerful technique that it is. First, it retains the benefits of a single-shot acquisition. For example, this enables application to in vivo diffusion-weighted imaging without the need for time-consuming navigators, as for instance required in 3D slab-selective DWI. In BOLD fMRI, physiological noise and motion is effectively "frozen out" rather than resulting in blurring as it may conceivably do in 3D acquisitions, and if sampled at an adequately high rate it can be removed in subsequent processing. In arterial spin labelling, SMS-EPI increases the volume that can be covered within the limited available sampling window, while the singleshot nature maintains well-defined timing at each slice location and allows arterial transit time to be determined.

The second important characteristic of SMS is that it provides SNR efficient sampling and even SNR advantage over regular 2D imaging. The slice acceleration in SMS is performed by simultaneously exciting multiple slices at different locations and as such a larger volume of spins contributes to the subsequently received signal, while the sampling time (e.g. EPI readout duration) is the same as in a singleslice acquisition. Slice acceleration does therefore not, unlike in-plane EPI acceleration, require the omission of $\mathrm{k}$-space samples and there is hence no $\operatorname{sqrt}\left(R_{\mathrm{sms}}\right) \mathrm{SNR}$ penalty. In fact, there is typically a healthy net gain in SNR per unit time from SMS acquisition, since many more imaging volumes can now be acquired per unit time, which for example allow better diffusion fitting in dMRI through acquiring data at more diffusion encoded directions, or to provide more temporal data points and improve the accuracy and specificity of fMRI analysis. However, it is important to note that this SNR benefit does get tapered by the SNR 

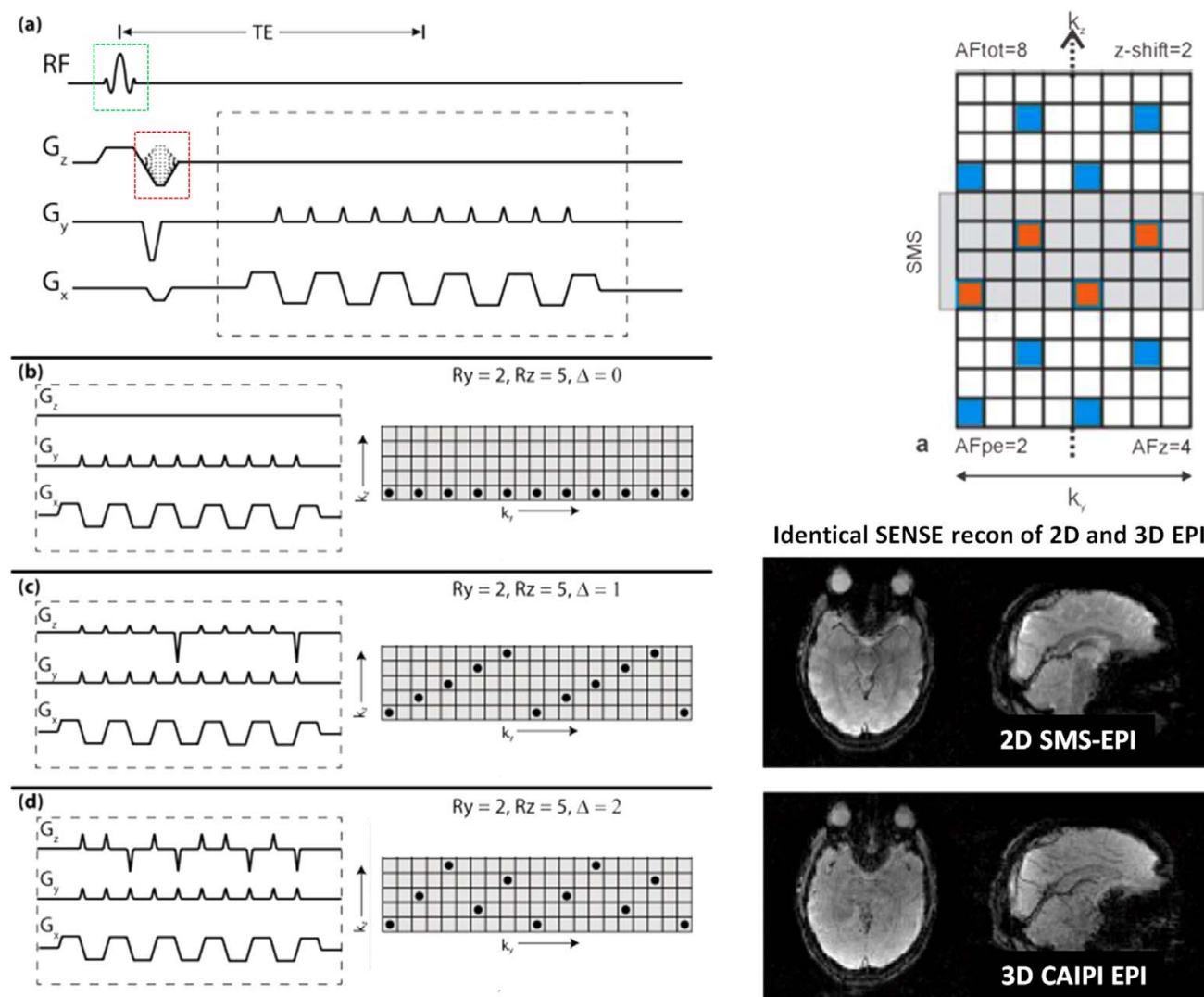

Identical SENSE recon of 2D and 3D EPI with $R y=2, R z=4$
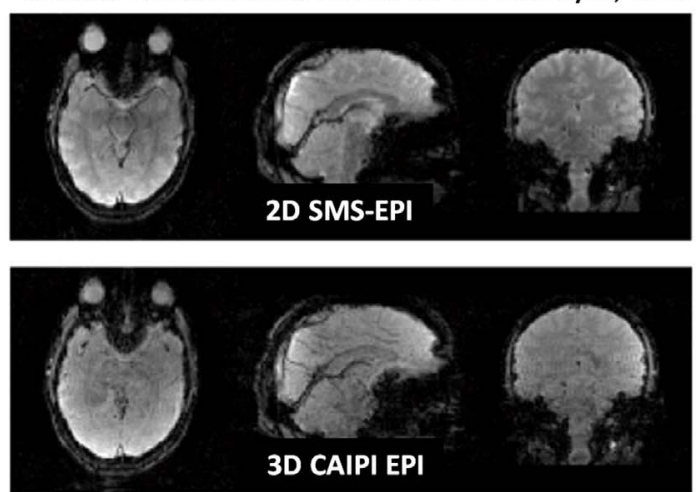

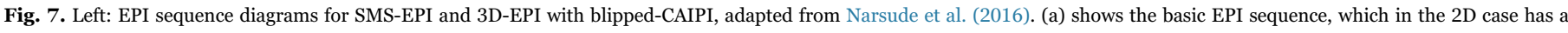

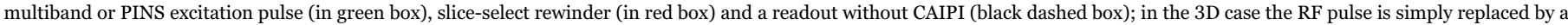

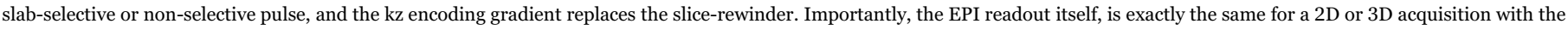

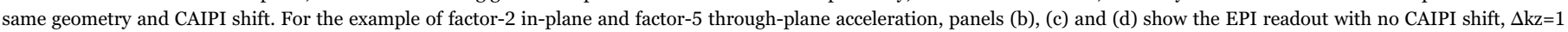

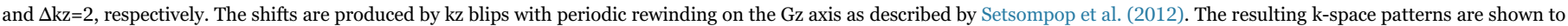

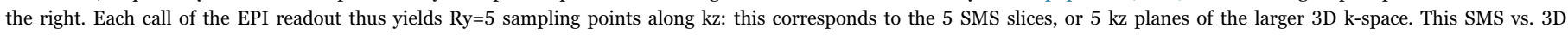

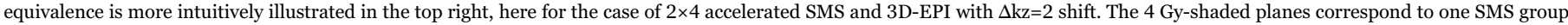

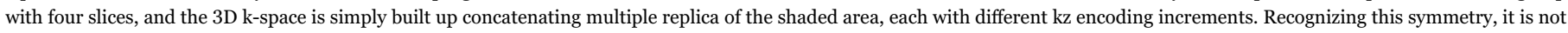

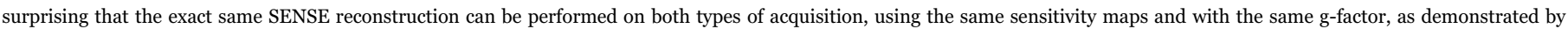
Zahneisen et al. (2015); the figures to the right are taken from that paper.

loss from the g-factor penalty and the reduced signal recovery of shorter TR. Nonetheless, these losses can be kept in check by selecting an acquisition protocol that does not push the slice acceleration to the limit.

In general there has been a trend in the move away from slice-byslice 2D imaging towards volumetric imaging with SMS and 3D acquisitions. The main benefits of volumetric imaging lie in the ability to achieve much higher accelerations through better utilization of coil sensitivity across more spatial dimensions with CAIPI, and in the large increase in SNR efficiency afforded from better signal averaging. In particular, 3D acquisitions can lead to an even higher SNR boost when compared to SMS acquisitions, since with every excitation and acquisition window data is now collected from all voxels in the imaging volume.

Recent development in CAIPI through the addition of the waveCAIPI method has enabled even higher accelerations in SMS and 3D acquisitions, allowing up to 15 fold acceleration with small g-factor noise penalty (Bilgic et al., 2015). Wave-CAIPI achieved this by extending the distribution of voxel aliasing of 2D-CAIPI from the phase encode and slice (or partition) directions to the read-out direction, to allow for a further increase in the distance between the aliasing voxels. This is realized by combining the $\mathrm{k}$-space undersampling pattern of 2D-CAIPIRINHA with a modified version of bunched phase encoding/zigzag sampling (Breuer et al., 2008; Moriguchi and Duerk, 2006) to play out sinusoidal (wave) gy and gz gradients during the data readout. Here, the sinusoidal wave gradients create a corkscrew k-space trajectory, which acts to spread the imaging voxels along the readout direction. This is illustrated in Fig. 8. It is interesting to note that a precursor to wave-CAIPI was actually proposed nearly decade ago in the thesis by Breuer (2008) through a simulation demonstrating that 2D-CAIPI with additional alternating $\Delta \mathrm{ky}$ and $\Delta \mathrm{kz}$ shifts during the readout can provide markedly improved PI performance.

The key steps forward in the recent wave-CAIPI development have been in an efficient parallel imaging reconstruction strategy for acquisitions with continuous and rapid varying wave gradients, and in an automatic gradient calibration method that can estimate gradient trajectory errors from system imperfection on the fly (Cauley et al., 2016). These developments have allowed robust, high quality waveCAIPI imaging to be performed at an order of magnitude undersampling for 3D and SMS imaging sequences of MPRAGE, SWI and T2-TSE as shown in Fig. 9. Here, the T2-TSE acquisition at SMS acceleration of 15 is achieved with low SAR through the use of MultiPINS refocusing pulses (Eichner et al., 2014b). It is expected that the high SNR and improved acceleration capability at UHF will allow imaging at even higher accelerations and resolutions than what is shown in Fig. 8 for $3 \mathrm{~T}$ acquisitions. We note that wave-CAIPI is ideally suited for low bandwidth acquisitions (e.g. 100-500 Hz) typically used in structural imaging, since the slow kx transversal in such cases allows large amount of wave encoding gradients to be played out. Nonetheless, it is expected that wave-CAIPI could still play an important role in improving the encoding efficiency of SMS-EPI and 3D-EPI acquisi- 


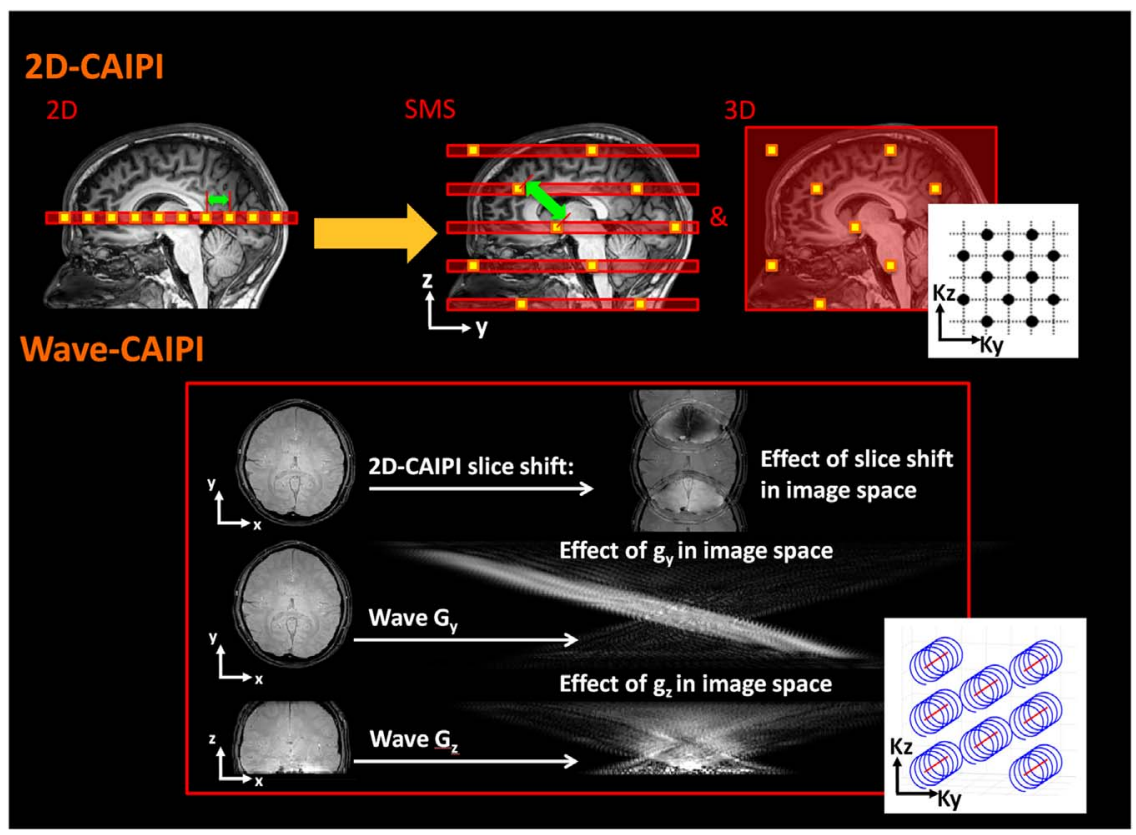

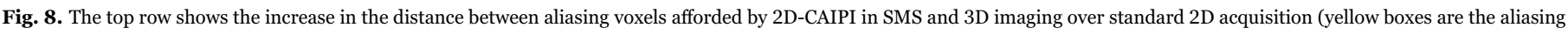

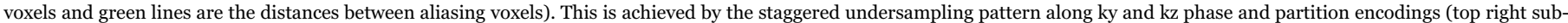

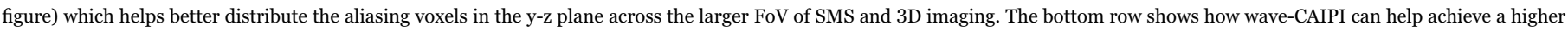

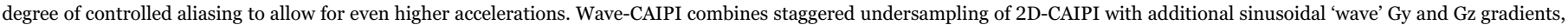

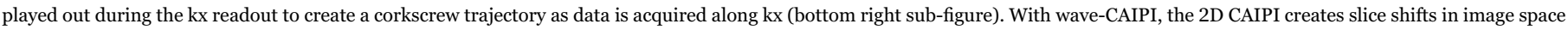

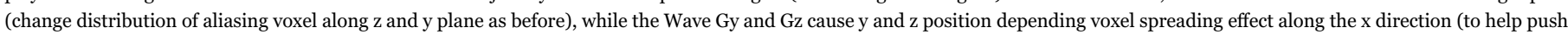

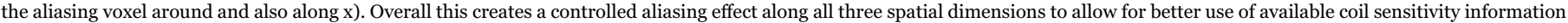
in all axes, in addition to the inherent acceleration benefit by more efficient sampling through k-space.

tions, in particular when using gradient systems with high slew rates, such as a head insert gradients.

\section{Key practical considerations}

As a general rule of thumb, the key criteria in selecting whether to use SMS or 3D acquisition lies in the application and contrast mechanism being used. Another consideration is the ability to tolerate or mitigate physiological noise and motion, which may be more pronounced in 3D acquisitions where more data are averaged together. On the other hand 3D encoding offers better-defined slice profile especially at high resolution, and the volume excitation makes it largely insensitive to motion-induced spin history effects. For contrast me- chanisms that require the use of long TRs, SMS acquisition is likely to be a good choice given that encoding of the other slices can be performed while waiting for the long TR, which allows for faster imaging. Examples here are SMS diffusion-weighted EPI or TSE structural imaging. On the other hand, if short TRs can be used and there is ability to tolerate or mitigate physiological noise and motion, then 3D imaging could prove a better choice that can provide higher SNR benefits. Prime examples of such cases are $\mathrm{T}_{2}{ }^{*}$-weighted scans for QSM or SWI, but also magnetization-prepared $\mathrm{T}_{1}$ weighted scans (MPRAGE, MP2RAGE) or magnetization-contrast enhanced scans.

In choosing an appropriate acceleration factor for a given acquisition, the reconstruction quality depends largely on the "total acceleration factor" $\left(\mathrm{R}_{\text {total }}=\mathrm{R}_{\text {inplane }} \times \mathrm{R}_{\text {slice }}\right)$; with higher $\mathrm{R}_{\text {total }}$ resulting in

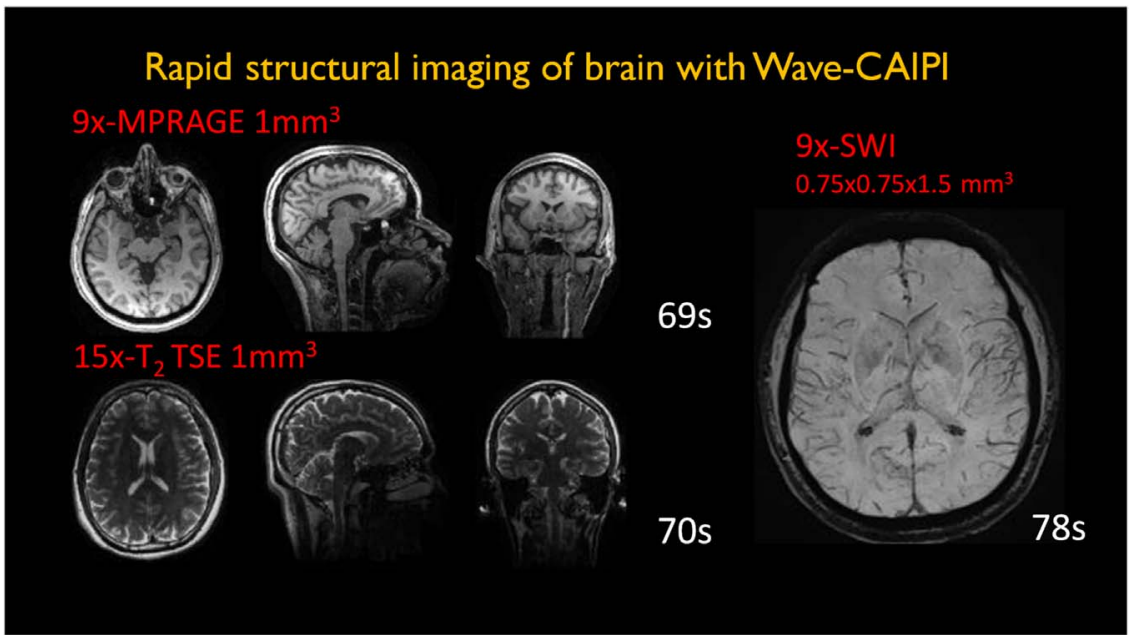

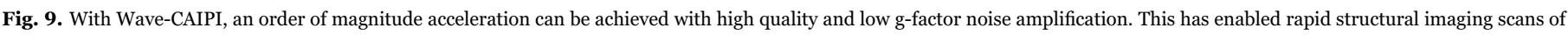

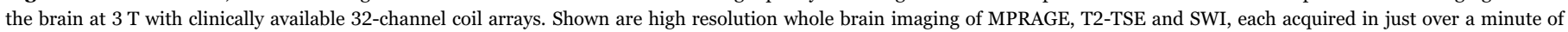
scanning. It is expected that the higher acceleration capability and the higher SNR of UHF will enable even better quality imaging, at higher resolution and faster imaging time. 
reduced reconstruction quality. Therefore, a tradeoff will need to be made between the amount of in-plane acceleration to mitigate image distortion and the amount of $\mathrm{R}_{\text {slice }}$ (slice acceleration in SMS-EPI, or partition acceleration in 3D-EPI), which provides faster imaging. For a 32-channel whole-brain coil array at $7 \mathrm{~T}$, total acceleration with CAIPI of $10-12 x$ is a good target for fMRI where thermal noise dominates and some g-factor noise is acceptable, while lower accelerations of 4-8x is good for SNR starved applications below approximately $1.5 \mathrm{~mm}$ isotropic. Note that the image quality and SNR typically experience a gradual decline with increasing acceleration, which is then followed by a rapid decline after reaching a total acceleration factor that is close to the available sensitivity encoding degree of freedom of the coil; for large channel count arrays this limit becomes increasingly independent of the exact coil geometry once a certain number of elements has been reached (Ohliger et al., 2003; Wiesinger et al., 2006). Note that the suggested acceleration factors given here are for whole-brain acquisition cases, and for SMS acquisition only applies for cases where at least a factor of $2-3 x$ slice acceleration is used to allow a large enough volumetric FoV coverage per acquisition to enable good controlled aliasing. For partial brain applications with smaller FoV, the distance between the aliased voxels will be smaller at the same acceleration factor, leading to a harder, more ill-conditioned reconstruction. Therefore, the total acceleration factor used should be scaled down accordingly. As a consequence, the much greater ability to accelerate acquisitions with large volume coverage diminishes the sampling time advantage of targeted small volume acquisitions that cannot achieve as high an acceleration factor.

For coil arrays with uniformly distributed coil elements (e.g. soccer ball geometry), the CAIPI shift factor/pattern should be chosen to help distribute the aliasing voxel uniformly across the imaging volume to maximize the distance between aliasing voxels. For other coil array designs, a CAIPI pattern that helps distribute the aliasing voxel more heavily along the direction(s) where there is (are) more coil sensitivity encoding information should be used. In most cases, a near-optimal choice of sampling pattern can be made by intuition or evaluation of the most suitable candidates, but full evaluation for the case of SMSEPI by simulation has also been described (Xu et al., 2013).

Subject motion can have a detrimental effect on accelerated acquisitions. Motion during reference data acquisition can lead to poor unalising performance for the whole time-series dataset. The use of motion-robust training data acquisition in GRAPPA based reconstructions, with e.g. FLEET or FLASH scan, can help mitigate such issues as has been shown for both 2D (Baron and Beaulieu, 2016; Polimeni et al., 2016; Talagala et al., 2013) and 3D-EPI (Ivanov et al., 2015). Motion during the time-series acquisition can also affect unaliasing performance since the underlying coil sensitivity information used for voxel unaliasing will now differ in the training and in the acquisition datasets. In particular, this issue can lead to large unaliasing artifacts when the eye regions with high signal intensity happens to be right at the edge of the imaging FoV, causing it to enter and exit the imaging FoV intermittently with small motions. Therefore, when possible, it is good practice to select the imaging FoV such that the eyes regions are either fully included or excluded from the FoV, with some spatial margins to allow for possible motions.

The use of high slice acceleration factor in SMS acquisitions can lead to RF pulses with high peak voltage. VERSE (Conolly et al., 1988) is typically used to mitigate this issue (at a cost of some distortion in the slice profile), to keep peak voltage to within the system hardware limit. However, for a large subject/patient, higher voltage could be needed and if the RF pulse was not designed properly to account for this (i.e. not VERSE-ed enough), RF clipping can occur, causing truncation of the RF pulse shape, which in turn can result in poor slice selection and image quality. In practice these restrictions are almost inevitably encountered in case of high-flip angle applications like dMRI which relies on 180 degree pulses, in practice limiting dMRI at $7 \mathrm{~T}$ to an SMS factor of 2 or maximally 3 (Eichner et al., 2014a; Vu et al., 2015). For BOLD fMRI with GE-EPI this is fortunately not always as limiting, because the much shorter TR that results from high SMS factors requires lower flip angles and hence peak voltage limitations do not apply as quickly as for high-flip angle scans. The BOLD experiment is by its nature is more forgiving to the choice of smallerthan-optimal excitation flip angle. This is especially the case if the fMRI experiment is performed in the physiological noise dominated regime (Gonzalez-Castillo et al., 2011), however at UHF it is usually desired to acquire high-resolution data in the image noise dominated regime in which case an effort should be made to match the Ernst angle as closely as possible to maximize image and hence temporal SNR.

\section{Public availability of advanced sequences and reconstruction methods}

While the data shown in publications on novel sampling schemes is typically reconstructed offline (e.g. in Matlab), the availability of sequences and reconstruction implementations on the major scanner platforms has rapidly increased in the past few years. SMS-EPI and volumetric CAIPIRINHA reconstruction is now offered commercially by the main vendors, and various application specific implementations are available as works-in-progress/collaborative packages, or patches from the vendors or research sites that offer their sequences to other labs. At time of writing, 'public' availability for Siemens platforms (as product, WIP, or C2P) includes SMS-EPI and 3D-EPI for BOLD fMRI, ASL, VASO and diffusion, as well as fast wave-CAIPI implementations of 3D GRE and 3D MPRAGE. Also Philips now have SMS product support for $\mathrm{fMRI}$ and diffusion sequences on their clinical systems and is extending the "MultiBand SENSE" framework to other 2D sequences and anatomies outside the brain, e.g. whole body diffusion as a worksin-progress package. Similarly, GE will be supporting the SMS feature (known as HyperBand on their platform) into its EPI based sequences for use in fMRI and diffusion applications. This list is rapidly growing. The speed at which new developments become available and the recently much greater active user interest in adapting new techniques is putting considerable but healthy pressure on the vendors to bring out high-quality commercial implementations.

\section{Tackling $\mathrm{B}_{1}{ }^{+}$-inhomogeneity and SAR}

$\mathrm{B}_{1}{ }^{+}$inhomogeneity and SAR limit our ability to fully realize the capability of UHF MRI, particularly for imaging sequences which utilize large flip-angle refocusing and/or inversion pulses. $\mathrm{B}_{1}{ }^{+}$inhomogeneity leads to non-uniformity in signal and more importantly contrast profiles, while SAR restrictions can demand inefficient acquisitions with large dead-times. These problems are long-standing and have been well recognized since the early days of UHF developments. Attractive mitigation solutions based on parallel transmission (pTx) have in fact been developed and demonstrated for nearly a decade now, but have been slow to gain traction due to widespread lack of user friendly scanner integrations. The main issue has been in a cumbersome workflow which requires lengthy $B_{1}$ and $B_{0}$ mapping, $R F$ pulse calculation and the use of careful on-the fly SAR management based on robust and safe, yet practical approaches to global and local SAR calculations. As none of the major vendors have yet provided a sufficiently useful set of tools for the design of pulses, let alone their integration into sequences, considerable engineering burden was thus left on the shoulders of researchers working on pTX. These issues have consequently held back pTx from being used in neuroscientific and clinical studies. Nonetheless, some important progress has been made to mitigate these practical obstacles, by devising more integrated workflows for $\mathrm{B}_{1}{ }^{+}$shimming and pulse designs. $\mathrm{B}_{1}{ }^{+}$mitigating pTx pulses are now starting to be demonstrated and used more often in imaging sequences such as MPRAGE and TSE, where clear benefits can be observed. For 3D imaging, good $\mathrm{B}_{1}{ }^{+}$mitigation can be achieved using short durations (2-3 ms) ' $\mathrm{k}_{\mathrm{T}}$-points' (Cloos et al., 2012) and spiral nonselective ('SPINS') (Malik et al., 2012) pulses. For 2D 
imaging, the 'Spokes' RF pulse (Saekho et al., 2006; Setsompop et al., 2008) has been widely used to simultaneously perform slice selection and in-plane $\mathrm{B}_{1}{ }^{+}$mitigation, and the extension to SMS has recently been demonstrated by different groups (Gras et al., 2016b; Poser et al., 2014; Sbrizzi et al., 2015; Wiggins 2016a; Wu et al., 2013). The Spoke design is however too lengthy for many applications and requires undesirable trade-offs between $\mathrm{B}_{1}{ }^{+}$mitigation, $\mathrm{B}_{0}$ robustness, slice profile and SAR. Adding to this issue is the high SAR of SMS pulses in general which is becoming more and more problematic with the recent push towards higher slice accelerations achieved through new CAIPI developments, particularly at UHF.

Of particular note in the context of SAR efficient SMS excitation are the aforementioned PINS (Norris et al., 2011) and multi-PINS pulses (Eichner et al., 2014b). The pulses result in a comb of slices being excited, and most importantly the required RF power is essentially independent of the number of effectively excited slices. This unique property makes the pulses particularly attractive for application in SMS spin-echo sequences such as the TSE examples shown in Fig. 5, and the wave-CAIPI accelerated TSE in Fig. 9 with up to factor-15 slice acceleration that would be impossible to achieve with regular multiband excitations. The gradient modulation during the pulses however results in somewhat longer pulses and hence reduced time-bandwidth product, making sharp slice profiles harder to achieve than for instance with regular sinc pulses. PINS pulses do not readily lend themselves to slice-by-slice $\mathrm{B}_{1}{ }^{+}$mitigation as for instance in SMS-spokes, since all slices in PINS are being excited by a single-band pulse. Efforts in this directions have nevertheless been made with $\mathrm{k}_{\mathrm{T}}$-PINS (Sharma et al., 2013), but the resulting pulses are impractically long for most applications. Therefore, $\mathrm{B}_{1}{ }^{+}$mitigation is in many ways still an uncracked problem that needs further research and innovation.

To this end, the 2016 ISMRM RF pulse design challenge (Grissom et al., 2016) was organized as a response to these issues, to i) find a better design for large-flip-angle, high slice-acceleration SMS pulses and ii) to develop better pTx pulses for $\mathrm{B}_{1}{ }^{+}$mitigated slice(s) selective excitation. The winning design for the large-flip-angle SMS pulse challenge (by rfcontrol team; Armin Rund, Christoph Aigner, and Christian Clason) was a new time-optimal control multiband pulse that achieve $5.1 \mathrm{x}$ shorter pulse duration than conventional approach (while keeping to within the SAR constraints), while the winning design for the pTx challenge (by StanfordUHF team; Mihir Pendse) was a new spokes pulse that combines VERSE with efficient SAR enforcing method to achieve 10.6x shorter pulse duration. These exciting developments should provide more head room for further accelerations and refinements of data acquisition at UHF.

After years spent on highly complex and typically slow pulse designs to squeeze the last bit of possible flip-angle homogenization, the pTx community has reached a turning point and recognizes that more practical solutions are required to bring pTx into routine application by lay users. One prime example is the recent concept of "universal pulses" (Gras et al., 2016a) which can be applied "blindly" to any typical brain without the need for subject-specific $\mathrm{B}_{1}{ }^{+}$and $\mathrm{B}_{0}$ mapping and pulse design, and yet achieve most of the possible improvement that a full subject-specific design would offer. This has been shown for the case of $\mathrm{k}_{\mathrm{T}}$-points but can in principle be applied to other pulses as well. Other pragmatic approaches include slice-by-slice 2D spokes pulse design in which, recognizing the slow spatial variation of $\mathrm{B}_{1}{ }^{+}$, a group of neighboring slices shares the same pulse to save computation time (Poser and Tse, 2015; Wu et al. 2016a, 2016b).

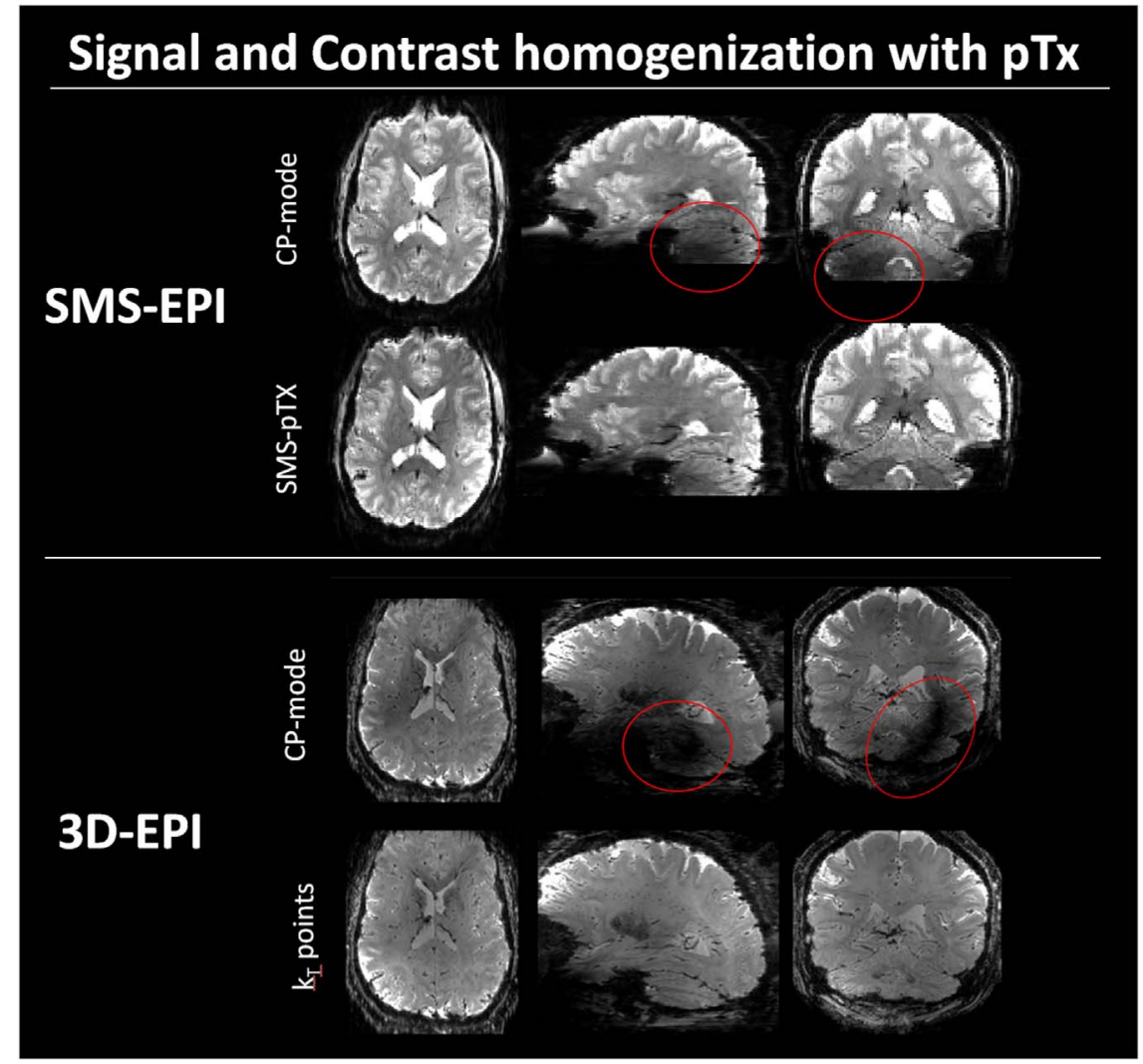

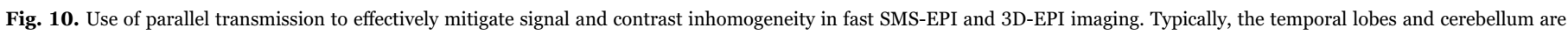

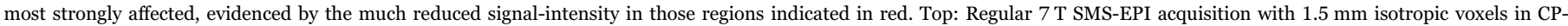

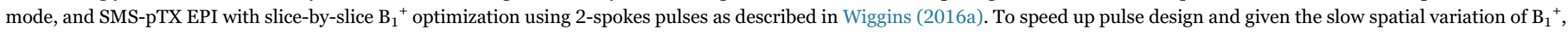

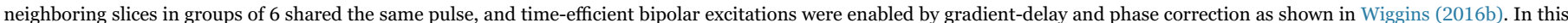

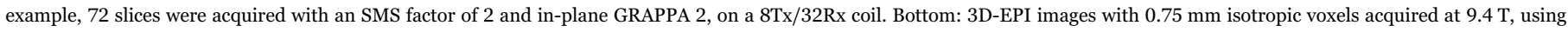

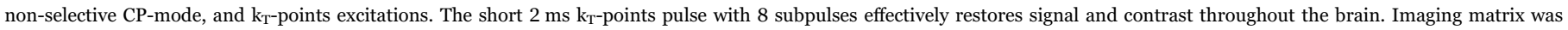
$256 \times 256 \times 244$ with $3 \times 2$ GRAPPA acceleration, on a $16 \mathrm{Tx} / 31 \mathrm{Rx}$ coil. The 3D-EPI images are taken from Poser et al. (2015). 
Fig. 10 shows illustrative examples of the improvements that pTx can bring to both SMS and volumetric acquisitions, shown here for the cases of SMS-EPI and 3D-EPI.

\section{Smart encoding in more dimensions}

The use of SNR-efficient volumetric acquisition via SMS and full 3D imaging along with the use of CAIPI to provide high parallel imaging accelerations have pushed the spatiotemporal resolution of UHF MRI to a level that would have seemed out of reach just a few years ago. A direction that can enable higher accelerations still, is in the incorporation of volume selective acquisition. In particular, the ZOOPPA approach (Heidemann et al., 2012) which combines zoomed imaging (via outer volume suppression) with parallel imaging has been shown to provide impressive accelerations, with the total acceleration $R_{\text {total }}=R_{\text {zoom }} \times R_{\text {inplane }} \times R_{\text {slice }}$. Such approach has also been modified to allow whole-brain coverage with reduced $\mathrm{FoV}_{\mathrm{PE}}$, (sag-ZOOPPA, to reduce distortion for EPI type acquisitions), which is achieved through sagittal scanning with saturation applied to the neck region and phase encoding performed in the head-foot direction. Moreover, the gradient-and-spin-echo (GRASE) sequences (Oshio and Feinberg, 1991) also provides an ability to utilize Inner Volume Imaging (IVI), to zoom the acquisition in two directions in order to target a small brain region and provide fast $3 \mathrm{D}$ encoding of this region at high spatial resolution (Feinberg et al., 2008). Nonetheless, zoomed imaging via outer volume suppression acts only to decrease the 'number' of aliasing voxels for a given $\mathrm{R}_{\text {total }}$ acceleration, but such approach does not decrease the distance between the aliasing voxels. Therefore the g-factor noise reduction benefit can be somewhat limited. An alternative approach in volume selective acquisition, which can actually increase the distance

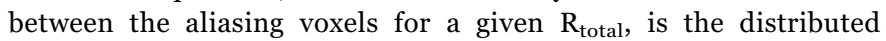
volumes selection and acquisition (Finsterbusch, 2015; Mooiweer et al., 2016). Fig. 11 shows an example where pTx is employed to provide 2D volume selective excitation, here in two apart brain regions (Poser et al., 2017). Another example of volume selective acquisition is in a modified 3D-GRASE, where the use of multiple intersecting RF slabs created by orthogonal MB slab-selective excitation pulses and orthogonal single-band refocusing pulses has enabled Simultaneous Multi-Volume imaging (Chen and Feinberg, 2013; Vu et al., 2013).

With these developments, the spatial encoding efficiency/acceleration that can be achieved in each imaging volume is likely to be close to the limit, and there is a need to look towards other degree of freedom in other dimensionality of encoding, to come up with the next large gain in acquisition speed. A promising direction of development has been in Magnetic Resonance Fingerprinting (MRF) (Ma et al., 2013), which has been built as a smart encoding approach for rapid multi-parameter mapping. Here the acquisition is designed to achieve incoherent aliasing in the space, time and contrast dimensions through the use of pseudo-random TR and flip-angle trains and variable density spiral acquisition. Such approach has enabled rapid mapping of multiple quantitative parameters (i.e. $\mathrm{T}_{1}, \mathrm{~T}_{2}$, proton density and $\mathrm{B}_{0}$ ) in a time frame of $10 \mathrm{~s} /$ slice, which is much faster than previously proposed methods. Recent developments in SMS techniques for MRF (SMS-MRF) have also helped further increase MRF acquisition efficiency by another 3-4 fold (Cloos et al., 2016b; Jiang et al., 2016; Ye et al., 2016, 2015). Moreover, the MRF framework has also been extended to create a powerful new idea in exploiting $\mathrm{B}_{1}{ }^{+}$inhomogeneity as an extra degree of freedom for data encoding rather than viewing it as a source of artifacts (Cloos et al., 2016a, 2014). Here, the inhomogeneous RF excitation modes of a multichannel transmit coil are interweavingly used during MRF acquisition to enable accurate quantitative parameter mapping in the present of wildly inhomogeneous $\mathrm{B}_{1}{ }^{+}$fields. Such an approach could be an ideal way in fully harnessing the high SNR and the inhomoge- neous $\mathrm{B}_{1}{ }^{+}$field at UHF to efficiently map quantitative MR parameters at a high spatial resolution.

Another active area of research has been in the development of smarter spatial-temporal encoding and reconstruction methods. In particular, exciting work is being performed in estimating and enforcing temporal low rank subspace for a number of MR acquisitions. Examples of some of these recent works include: i) $\mathrm{T}_{2}$ shuffling method for 3D-FSE which fixes the image blurring of FSE while providing full images at multiple TEs from a single efficient acquisition (Tamir et al., 2016), ii) SPICE acquisition that utilizes subspace modeling to boost the speed and SNR of MRSI (Lam et al., 2016; Ma et al., 2016), and iii) k-t FASTER approach which make use of low rank truncation to accelerate fMRI acquisitions (Chiew et al., 2016, 2015). A common and important theme in all these techniques in achieving good performance has been in the careful design of both the acquisition and reconstruction that can fully exploit the low rank modeling that is being used. Just as CAIPI fully exploits additional spatial encoding dimensions in parallel imaging to provide large multiplicative gains in accelerations, the complimentary use of these low rank approaches along time/ contrast dimensions should provide exciting opportunities for further large gains in encoding efficiency. In combining these emerging acquisition approaches with the SNR/CNR benefits of UHF, we should be able to achieve very high quality, high spatiotemporal resolution imaging that could aid in propelling the MRI field forward.

\section{Summary, outlook}

The rapidly increasing availability of UHF scanners has created the opportunity and desire to perform acquisitions at spatial resolutions that are not feasible at clinical field strengths and may have been thought impossible only a few years ago. In particular, the gains in SNR and CNR brought about by UHF are typically translated (at least partially) into smaller voxel sizes and hence larger acquisition matrices, which creates a huge encoding and reconstruction challenge. The skyrocketing increase in measurement time that would be required to perform the encoding of large matrices with standard techniques, would more often than not render the endeavor impractical for in vivo application: unacceptably long TR in fMRI, or unacceptably long scan time for structural imaging like multi-directional QSM. There is hence great demand for new smart acquisition strategies that more fully exploit the encoding power offered by the modern day high-channelcount RF receive coils. New directions that evolved at breathtaking pace during the past few years are the highly accelerated SMS and 3D acquisitions with controlled aliasing which have enabled large speedup factors without excessive g-noise penalties. This technology has now matured to a degree that researchers can perform a (whole-brain) fMRI acquisition at factor 12 undersampling without too much hesitation. In parallel, other developments are being worked on the hardware side, including massively parallel receive hardware, improved gradient coils or new strategies for local or dynamic B0 shimming. In addition, after years of being regarded as a 'promising technique' but too clunky for real use, parallel RF transmission is now making its entry into first routine applications, enabling more of the UHF potentials to be harnessed but also potentially play an increasing role in the encoding itself, as well. MRI sampling strategies that continue to combine elements of the various advances in new ways, are expected to enable further accelerations well beyond what is achievable now. In particular we need to be prepared for other paradigm shifts, eg. the acceptance of MR finger-printing or new encoding strategies that, exploit the temporal/multi-contrast signal sparsity.

\section{Acknowledgements}

We thank our colleagues and collaborators for contributing images and data to this paper and being available for discussion. Thanks to Dr Dimo Ivanov for scan support, Dr Desmond Tse for the pTX work and 

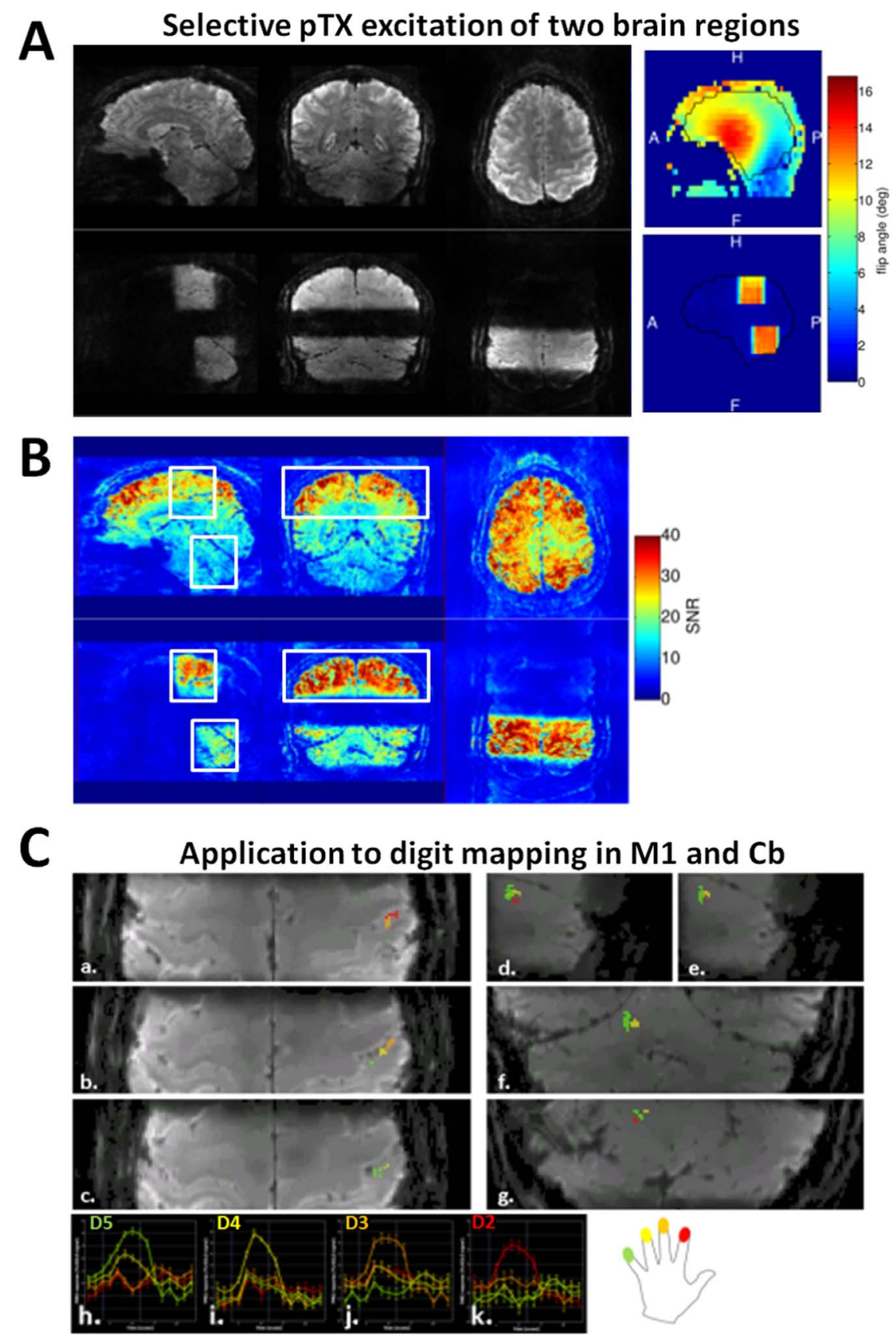

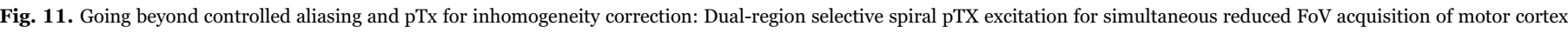

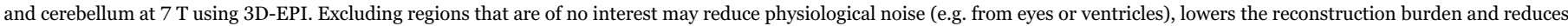

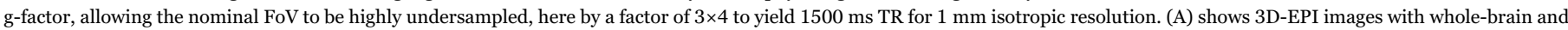

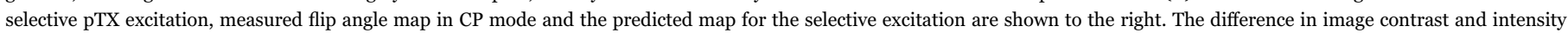

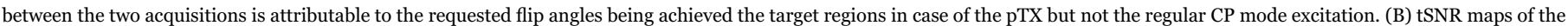

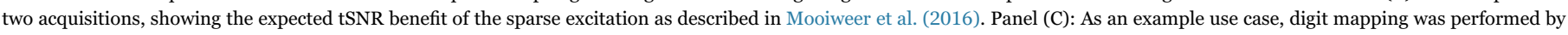

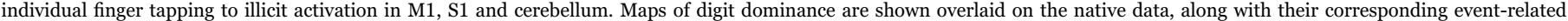
BOLD response. Images are taken from Poser et al. (2017).

Dr Chris Wiggins for hardware support. Also, thanks to Dr Berkin Bilgic, Dr Stephen F. Cauley and Dr Jonathan Polimeni for the blippedCAIPI, wave-CAIPI and TOF-MRA work. Maastricht scan time was funded by intramural MBIC funding and Scannexus/BrainsUnlimited project dev_b0b1. Dr Poser was partly funded by R01MH111444 (PI Feinberg). Dr Setsompop's effort was supported in part by the NIH National Institute for Biomedical Imaging and Bioengineering (P41EB015896, R01-EB019437 and R01-EB020613) and by the NIH Brain Initiative project R24MH106096.

\section{References}

Baron, C.A., Beaulieu, C., 2016. Motion robust GRAPPA for echo-planar imaging. Magn. Reson. Med. 75, 1166-1174. http://dx.doi.org/10.1002/mrm.25705.

Barth, M., Meyer, H., Kannengiesser, S.A.R., Polimeni, J.R., Wald, L.L., Norris, D.G., 2010. $\mathrm{T}_{2}$-weighted 3D fMRI using $\mathrm{S}_{2}$-SSFP at $7 \mathrm{~T}$. Magn. Reson. Med. 63, 1015-1020. http://dx.doi.org/10.1002/mrm.22283.

Barth, M., Breuer, F., Koopmans, P.J., Norris, D.G., Poser, B.A., 2015. Simultaneous multislice (SMS) imaging techniques. Magn. Reson. Med. 81. http://dx.doi.org/ $10.1002 / \mathrm{mrm} .25897$.

Bause, J., Ehses, P., Mirkes, C., Shajan, G., Scheffler, K., Pohmann, R., 2016. Quantitative 
and functional pulsed arterial spin labeling in the human brain at 9.4 t. Magn. Reson. Med. 75, 1054-1063. http://dx.doi.org/10.1002/mrm.25671.

Bilgic, B., Pfefferbaum, A., Rohlfing, T., Sullivan, E.V., Adalsteinsson, E., 2012. MRI estimates of brain iron concentration in normal aging using quantitative susceptibility mapping. Neuroimage 59, 2625-2635. http://dx.doi.org/10.1016/ j.neuroimage.2011.08.077.

Bilgic, B., Gagoski, B.A., Cauley, S.F., Fan, A.P., Polimeni, J.R., Grant, P.E., Wald, L.L., Setsompop, K., 2015. Wave-CAIPI for highly accelerated 3D imaging. Magn. Reson. Med. 73, 2152-2162. http://dx.doi.org/10.1002/mrm.25347.

Bilgic, B., Xie, L., Dibb, R., Langkammer, C., Mutluay, A., Ye, H., Polimeni, J., Augustinack, J., Liu, C., Wald, L., Setsompop, K., 2016. Rapid multi-orientation quantitative susceptibility mapping. Neuroimage 125, 1131-1141.

Blaimer, M., Breuer, F. a, Seiberlich, N., Mueller, M.F., Heidemann, R.M., Jellus, V., Wiggins, G., Wald, L.L., Griswold, M. a, Jakob, P.M., 2006. Accelerated volumetric MRI with a SENSE/GRAPPA combination. J. Magn. Reson. imaging 24, 444-450. http://dx.doi.org/10.1002/jmri.20632.

Breuer, F.A., 2008. Development and Application of Efficient Strategies for Parallel Magnetic Resonance Imaging. Julius-Maximilians-Universität Würzburg, Würzburg, Germany/https://opus.bibliothek.uni-wuerzburg.de/files/1760/thesis_breuer.pdf〉.

Breuer, F.A., Blaimer, M., Heidemann, R.M., Mueller, M.F., Griswold, M.A., Jakob, P.M., 2005. Controlled aliasing in parallel imaging results in higher acceleration (CAIPIRINHA) for multi-slice imaging. Magn. Reson. Med. 53, 684-691. http:// dx.doi.org/10.1002/mrm.20401.

Breuer, F.A., Blaimer, M., Mueller, M.F., Seiberlich, N., Heidemann, R.M., Griswold, M.A., Jakob, P.M., 2006. Controlled aliasing in volumetric parallel imaging (2D CAIPIRINHA). Magn. Reson. Med. 55, 549-556. http://dx.doi.org/10.1002/ mrm.20787.

Breuer, F.A., Moriguchi, H., Seiberlich, N., Blaimer, M., Jakob, P.M., Duerk, J.L. Griswold, M.A., 2008. Zigzag sampling for improved parallel imaging. Magn. Reson. Med. 60, 474-478. http://dx.doi.org/10.1002/mrm.21643.

Buxton, R.B., Wong, E.C., Frank, L.R., 1998. Dynamics of blood flow and oxygenation changes during brain activation: the ballon model. Magn. Reson. Med. 39, 855-864. http://dx.doi.org/10.1002/mrm.1910390602.

Cauley, S.F., Polimeni, J.R., Bhat, H., Wald, L.L., Setsompop, K., 2013. Interslice leakage artifact reduction technique for simultaneous multislice acquisitions. Magn. Reson. Med. 0, 1-10. http://dx.doi.org/10.1002/mrm.24898.

Cauley, S.F., Setsompop, K., Bilgic, B., Bhat, H., Gagoski, B., Wald, L.L., 2016. Autocalibrated wave-CAIPI reconstruction; Joint optimization of k-space trajectory and parallel imaging reconstruction. Magn. Reson. Med.. http://dx.doi.org/10.1002/ mrm.26499.

Chen, L., Feinberg, D.A., 2013. Simultaneous Multi-Volume GRASE Imaging. Proc. Int. Soc. Mag. Reson. Med. 21, 2365.

Chiew, M., Smith, S.M., Koopmans, P.J., Graedel, N.N., Blumensath, T., Miller, K.L., 2015. K-t FASTER: acceleration of functional MRI data acquisition using low rank constraints. Magn. Reson. Med. 74, 353-364. http://dx.doi.org/10.1002/ mrm.25395.

Chiew, M., Graedel, N.N., Mcnab, J.A., Smith, S.M., Miller, K.L., 2016. Accelerating functional MRI using fixed-rank approximations and radial-cartesian sampling. Magn. Reson. Med.. http://dx.doi.org/10.1002/mrm.26079.

Cloos, M.A., Boulant, N., Luong, M., Ferrand, G., Giacomini, E., Le Bihan, D., Amadon, a, 2012. kT -points: short three-dimensional tailored RF pulses for flip-angle homogenization over an extended volume. Magn. Reson. Med. 67, 72-80. http:// dx.doi.org/10.1002/mrm.22978.

Cloos, M.A., Wiggins, C., Wiggins, G., Sodickson, D., 2014. Plug and play parallel transmission at 7 and 9.4 T based on principles from MR fingerprinting. Proc. Int. Soc. Mag. Reson. Med., 542.

Cloos, Knoll, F., Zhao, T., Block, K.T., Bruno, M., Wiggins, G.C., Sodickson, D.K., 2016a. Multiparametric imaging with heterogeneous radiofrequency fields. Nat. Commun. 7,12445 .

Cloos, Zhao, T., Knoll, F., Sodickson, D.K., 2016b. Online Radial Multiband Magnetic Resonance Fingerprinting. In: Proceedings International Soc Mag Reson Med. p. 608.

Conolly, S., Nishimura, D., Macovski, A., Glover, G., 1988. Variable-rate selective excitation. J. Magn. Reson. 78, 440-458. http://dx.doi.org/10.1016/0022-2364(88) 90131-X.

Cox, E., Gowland, P., 2008. Measuring T2 and T2' in the brain at $1.5 \mathrm{~T}, 3 \mathrm{~T}$ and $7 \mathrm{~T}$ using a hybrid gradient echo-spin echo sequence and EPI. In: Proceedings International Soc. Mag. Reson. Med.

Curtis, A.T., Hutchison, R.M., Menon, R.S., 2014. Phase based venous suppression in resting-state BOLD GE-fMRI. Neuroimage 100, 51-59. http://dx.doi.org/10.1016/ j.neuroimage.2014.05.079.

Dale, A.M., Fischl, B., Sereno, M.I., 1999. Cortical Surface-based analysis: i. Segmentation and surface reconstruction. Neuroimage 9, 179-194. http:// dx.doi.org/10.1006/nimg.1998.0395.

De Martino, F., Moerel, M., van de Moortele, P.-F., Ugurbil, K., Goebel, R., Yacoub, E., Formisano, E., 2013a. Spatial organization of frequency preference and selectivity in the human inferior colliculus. Nat. Commun. 4, 1386. http://dx.doi.org/10.1038/ ncomms2379.

De Martino, F., Zimmermann, J., Muckli, L., Uğurbil, K., Yacoub, E., Goebel, R., 2013b. Cortical depth dependent functional responses in humans at 7T: improved specificity with 3D GRASE. PLoS One 8, e60514.

Deistung, A., Rauscher, A., Sedlacik, J., Stadler, J., Witoszynskyj, S., Reichenbach, J.R., 2008. Susceptibility weighted imaging at ultra high magnetic field strengths: theoretical considerations and experimental results. Magn. Reson. Med. 60, 1155-1168. http://dx.doi.org/10.1002/mrm.21754.

Detre, J.A., Leigh, J.S., Williams, D.S., Koretsky, A.P., 1992. Perfusion Imaging. Magn.
Reson. Med. 23, 37-45. http://dx.doi.org/10.1002/mrm.1910230106.

Devor, A., Sakadžić, S., Srinivasan, V.J., Yaseen, M.A., Nizar, K., Saisan, P.A., Tian, P., Dale, A.M., Vinogradov, S.A., Franceschini, M.A., Boas, D.A., 2012. Frontiers in optical imaging of cerebral blood flow and metabolism. J. Cereb. Blood Flow. Metab. 32, 1259-1276. http://dx.doi.org/10.1038/jcbfm.2011.195.

Donahue, M.J., Blicher, J.U., Østergaard, L., Feinberg, D.A., MacIntosh, B.J., Miller, K.L., Günther, M., Jezzard, P., 2009. Cerebral blood flow, blood volume, and oxygen metabolism dynamics in human visual and motor cortex as measured by whole-brain multi-modal magnetic resonance imaging. J. Cereb. Blood Flow Metab. 29, 1856-1866. http://dx.doi.org/10.1038/jcbfm.2009.107.

Duyn, J.H., van Gelderen, P., Li, T.-Q., de Zwart, J. a, Koretsky, A.P., Fukunaga, M., 2007. High-field MRI of brain cortical substructure based on signal phase. Proc. Natl. Acad. Sci. USA 104, 11796-11801. http://dx.doi.org/10.1073/ pnas.0610821104.

Eichner, C., Setsompop, K., Koopmans, P.J., Lützkendorf, R., Norris, D.G., Turner, R., Wald, L.L., Heidemann, R.M., 2014a. Slice accelerated diffusion-weighted imaging at ultra-high field strength. Magn. Reson. Med. 71, 1518-1525. http://dx.doi.org/ 10.1002/mrm.24809.

Eichner, C., Wald, L.L., Setsompop, K., 2014b. A low power radiofrequency pulse for simultaneous multislice excitation and refocusing. Magn. Reson. Med. 72, 949-958. http://dx.doi.org/10.1002/mrm.25389.

Feinberg, D.A., Harel, N., Ramanna, S., Uğurbil, K., Yacoub, E., 2008. Sub-millimeter single-shot 3D GRASE with inner volume selection for T2-weighted fMRI applications at 7 T. Proc. Int. Soc. Mag. Reson. Med 16, 2373.

Feinberg, D.A., Moeller, S., Smith, S.M., Auerbach, E., Ramanna, S., Glasser, M.F., Miller, K.L., Ugurbil, K., Yacoub, E., 2010. Multiplexed Echo Planar Imaging for subsecond whole brain fMRI and fast diffusion imaging. PLoS One 5, e15710. http:// dx.doi.org/10.1371/journal.pone.0015710.

Finsterbusch, J., 2015. Simultaneous functional MRI acquisition of distributed brain regions with high temporal resolution using a $2 \mathrm{D}$-selective radiofrequency excitation. Magn. Reson. Med. 73, 683-691. http://dx.doi.org/10.1002/mrm.25143.

Fischl, B., Dale, A.M., 2000. Measuring the thickness of the human cerebral cortex from magnetic resonance images. Proc. Natl. Acad. Sci. USA. 97, 11050-11055. http:// dx.doi.org/10.1073/pnas.200033797.

Fischl, B., Sereno, M.I., Dale, A.M., 1999. Cortical surface-based analysis: ii: inflation, flattening, and a surface-based coordinate system. Neuroimage 9, 195-207. http:// dx.doi.org/10.1006/nimg.1998.0396.

Fukunaga, M., Li, T.-Q., van Gelderen, P., de Zwart, J. a, Shmueli, K., Yao, B., Lee, J., Maric, D., Aronova, M. a, Zhang, G., Leapman, R.D., Schenck, J.F., Merkle, H., Duyn, J.H., 2010. Layer-specific variation of iron content in cerebral cortex as a source of MRI contrast. Proc. Natl. Acad. Sci. USA. 107, 3834-3839. http://dx.doi.org/ 10.1073/pnas.0911177107.

Gardener, A.G., Jezzard, P., 2015. Investigating white matter perfusion using optimal sampling strategy arterial spin labeling at 7 T. Magn. Reson. Med. 73, 2243-2248. http://dx.doi.org/10.1002/mrm.25333.

Gardener, A.G., Gowland, P.A., Francis, S.T., 2009. Implementation of quantitative perfusion imaging using pulsed arterial spin labeling at ultra-high field. Magn. Reson. Med. 61, 874-882. http://dx.doi.org/10.1002/mrm.21796.

Ghariq, E., Teeuwisse, W.M., Webb, A.G., van Osch, M.J.P., 2012. Feasibility of pseudocontinuous arterial spin labeling at $7 \mathrm{~T}$ with whole-brain coverage. Magn. Reson. Mater. Phys. 25, 83-93. http://dx.doi.org/10.1007/s10334-011-0297-0.

Glover, G.H., 1991. Phase-offset multiplanar (POMP) volume imaging: a new technique. J Magn. Reson. Imaging 1, 457-461. http://dx.doi.org/10.1002/jmri.1880010410.

Glover, G.H., Li, T.Q., Ress, D., 2000. Image-based method for retrospective correction of physiological motion effects in fMRI: RETROICOR. Magn. Reson. Med. 44, $162-167$.

Goa, P.E., Koopmans, P.J., Poser, B.A., Barth, M., Norris, D.G., 2014. Bold fMRI signal characteristics of S1- and S2-SSFP at 7 T. Front. Neurosci.. http://dx.doi.org/ 10.3389 /fnins.2014.00049.

Golay, X., Petersen, E.T., 2006. Arterial spin labeling: benefits and pitfalls of high magnetic field. Neuroimaging Clin. N. Am. 16, 259-268. http://dx.doi.org/10.1016/ j.nic.2006.02.003.

Gonzalez-Castillo, J., Roopchansingh, V., Bandettini, P.A., Bodurka, J., 2011. Physiological noise effects on the flip angle selection in BOLD fMRI. Neuroimage 54, 2764-2778. http://dx.doi.org/10.1016/j.neuroimage.2010.11.020.

Gras, V., Vignaud, A., Amadon, A., Le Bihan, D., Boulant, N., 2016a. Universal pulses: a new concept for calibration-free parallel transmission. Magn. Reson. Med.. http:// dx.doi.org/10.1002/mrm.26148.

Gras, V., Vignaud, A., Amadon, A., Mauconduit, F., Le Bihan, D., Boulant, N., 2016b. In vivo demonstration of whole-brain multislice multispoke parallel transmit radiofrequency pulse design in the small and large flip angle regimes at $7 \mathrm{~T}$. Magn. Reson. Med.. http://dx.doi.org/10.1002/mrm.26491.

Griffanti, L., Salimi-Khorshidi, G., Beckmann, C.F., Auerbach, E.J., Douaud, G., Sexton, C.E., Zsoldos, E., Ebmeier, K.P., Filippini, N., Mackay, C.E., Moeller, S., Xu, J., Yacoub, E., Baselli, G., Ugurbil, K., Miller, K.L., Smith, S.M., 2014. ICA-based artefact removal and accelerated fMRI acquisition for improved resting state network imaging. Neuroimage 95, 232-247. http://dx.doi.org/10.1016/ j.neuroimage.2014.03.034.

Grissom, W.A., Setsompop, K., Hurley, S.A., Tsao, J., Velikina, J.V., Samsonov, A.A., 2016. Advancing RF pulse design using an open-competition format: report from the 2015 ISMRM challenge. Magn. Reson. Med. 0, 1-10. http://dx.doi.org/10.1002/ mrm.26512.

Griswold, M.A., Jakob, P.M., Heidemann, R.M., Nittka, M., Jellus, V., Wang, J., Kiefer, B., Haase, A., 2002. Generalized autocalibrating partially parallel acquisitions (GRAPPA). Magn. Reson. Med. 47, 1202-1210. http://dx.doi.org/10.1002/ mrm.10171. 
Haacke, E.M., Xu, Y., Cheng, Y.-C.N., Reichenbach, J.R., 2004. Susceptibility weighted imaging (SWI). Magn. Reson. Med. 52, 612-618. http://dx.doi.org/10.1002/ mrm.20198.

Haacke, E.M., Mittal, S., Wu, Z., Neelavalli, J., Cheng, Y.-C.N., 2009. Susceptibilityweighted imaging: technical aspects and clinical applications, part 1. AJNR. Am. J. Neuroradiol. 30, 19-30. http://dx.doi.org/10.3174/ajnr.A1400.

Heidemann, R.M., Porter, D. a, Anwander, A., Feiweier, T., Heberlein, K., Knösche, T.R., Turner, R., 2010. Diffusion imaging in humans at $7 \mathrm{~T}$ using readout-segmented EPI and GRAPPA. Magn. Reson. Med. 64, 9-14. http://dx.doi.org/10.1002/mrm.22480.

Heidemann, R.M., Anwander, A., Feiweier, T., Knösche, T.R., Turner, R., 2012. k-space and q-space: combining ultra-high spatial and angular resolution in diffusion imaging using ZOOPPA at 7 T. Neuroimage 60, 967-978. http://dx.doi.org/ 10.1016/j.neuroimage.2011.12.081.

Hua, J., Jones, C.K., Qin, Q., Van Zijl, P.C.M., 2013. Implementation of vascular-spaceoccupancy MRI at 7T. Magn. Reson. Med. 69, 1003-1013. http://dx.doi.org/ $10.1002 / \mathrm{mrm} .24334$

Huber, L., Ivanov, D., Krieger, S.N., Streicher, M.N., Mildner, T., Poser, B.A., Möller, H.E., Turner, R., 2014. Slab-selective, BOLD-corrected VASO at $7 \mathrm{~T}$ provides measures of cerebral blood volume reactivity with high signal-to-noise ratio. Magn. Reson. Med. 72. http://dx.doi.org/10.1002/mrm.24916.

Huber, L., Ivanov, D., Guidi, M., Turner, R., Uludağ, K., Möller, H.E., Poser, B.A., 2016. Functional cerebral blood volume mapping with simultaneous multi-slice acquisition. Neuroimage 125. http://dx.doi.org/10.1016/j.neuroimage.2015.10.082.

Huber, Handwerker, D.A., Gonzalez-Castillo, J., Jangraw, D., Guidi, M., Ivanov, D.,

Poser, B.A., Goense, J., Bandettini, P.A., 2016a. Effective connectivity measured with layer- dependent resting-state blood volume fMRI in humans. Proc. In. Soc. Magn. Reson. Med., 948. http://dx.doi.org/10.1002/mrm.25628.

Huber, Ivanov, D., Handwerker, D.A., Marrett, S., Guidi, M., Uludağ, K., Bandettini, P.A., Poser, B.A., 2016b. Techniques for blood volume fMRI with VASO: from lowresolution mapping towards sub-millimeter layer-dependent applications. Neuroimage. http://dx.doi.org/10.1016/j.neuroimage.2016.11.039.

Huber, Ivanov, D., Handwerker, D.A., Marrett, S., Guidi, M., Uludağ, K., Bandettini, P.A., Poser, B.A., 2016c. Techniques for blood volume fMRI with VASO: from lowresolution mapping towards sub-millimeter layer-dependent applications. Neuroimage. http://dx.doi.org/10.1016/j.neuroimage.2016.11.039.

Ivanov, D., Bart, M., Ulusag, K., Poser, B.A., 2015. Robust calibration data (ACS) acquisition for 3D echo planar imaging. In: ISMRM

Ivanov, D., Kashyap, S., Gardumi, A., Poser, B.A., Uludag, K., 2016a. Sub-millimeter human brain perfusion maps using arterial spin labelling at 3 and $7 \mathrm{~T}$. In: ISMRM Workshop on Ultra High Field MRI. p. 40.

Ivanov, D., Poser, B.A., Huber, L., Pfeuffer, J., Uludağ, K., 2016b. Optimization of simultaneous multislice EPI for concurrent functional perfusion and BOLD signal measurements at 7 T. Magn. Reson. Med.. http://dx.doi.org/10.1002/mrm.26351.

Jiang, Y., Ma, D., Bhat, H., Ye, H., Cauley, S.F., Wald, L.L., Setsompop, K., Griswold, M. A., 2016. Use of pattern recognition for unaliasing simultaneously acquired slices in Simultaneous MultiSlice Magnetic Resonance Fingerprinting. Magn. Reson. Med. early view.

Jin, T., Kim, S.-G.G., 2006. Spatial dependence of CBV-fMRI: A comparison between VASO and contrast agent based methods. In: Annual International Conference of the IEEE Engineering in Medicine and Biology - Proceedings. IEEE, pp. 25-28. 〈http:// dx.doi.org/10.1109/IEMBS.2006.259553>.

Jin, T., Kim, S.G., 2008. Improved cortical-layer specificity of vascular space occupancy fMRI with slab inversion relative to spin-echo BOLD at 9.4??T. Neuroimage 40, 59-67. http://dx.doi.org/10.1016/j.neuroimage.2007.11.045.

Kemper, V.G., Martino, F., De, Vu, A.T., Poser, B.A., Feinberg, D.A., Yacoub, E., Goebel, R., 2014. Evaluation of point spread function and functional sensitivity of 3DGRASE and 2D Spin-Echo EPI for sub-millimeter-resolution fMRI at 7 T. Proc. Int. Soc. Mag. Reson. Med. 22, 2987.

Kim, S.-G., Harel, N., Jin, T., Kim, T., Lee, P., Zhao, F., 2013. Cerebral blood volume MRI with intravascular superparamagnetic iron oxide nanoparticles. NMR Biomed. 26 949-962. http://dx.doi.org/10.1002/nbm.2885.

Koopmans, P.J., 2016. Two-dimensional-NGC-SENSE-GRAPPA for fast, ghosting-robust reconstruction of in-plane and slice-accelerated blipped-CAIPI echo planar imaging. Magn. Reson. Med.. http://dx.doi.org/10.1002/mrm.26179.

Koopmans, P.J., Manniesing, R., Niessen, W.J., Viergever, M. a, Barth, M., 2008. MR venography of the human brain using susceptibility weighted imaging at very high field strength. MAGMA 21, 149-158. http://dx.doi.org/10.1007/s10334-007-0101 3.

Koopmans, P.J., Barth, M., Norris, D.G., 2010. Layer-specific BOLD activation in human V1. Hum. Brain Mapp. 31, 1297-1304. http://dx.doi.org/10.1002/hbm.20936.

Koretsky, A.P., 2012. Early development of arterial spin labeling to measure regional brain blood flow by MRI. Neuroimage 62, 602-607. http://dx.doi.org/10.1016/ j.neuroimage.2012.01.005.

van der Kouwe, A.J.W., Benner, T., Salat, D.H., Fischl, B., 2008. Brain morphometry with multiecho MPRAGE. Neuroimage 40, 559-569. http://dx.doi.org/10.1016/ j.neuroimage.2007.12.025.

Lam, F., Ma, C., Clifford, B., Johnson, C.L., Liang, Z.-P., 2016. High-resolution (1) HMRSI of the brain using SPICE: data acquisition and image reconstruction. Magn. Reson. Med. 76, 1059-1070. http://dx.doi.org/10.1002/mrm.26019.

Larkman, D.J., Hajnal, J.V., Herlihy, A.H., Coutts, G.A., Young, I.R., Ehnholm, G., 2001 Use of multicoil arrays for separation of signal from multiple slices simultaneously excited. J. Magn. Reson. Imaging 13, 313-317.

Lee, H.-L., Zahneisen, B., Hugger, T., LeVan, P., Hennig, J., 2013. Tracking dynamic resting-state networks at higher frequencies using MR-encephalography. Neuroimage 65, 216-222. http://dx.doi.org/10.1016/j.neuroimage.2012.10.015.

Lewis, L.D., Setsompop, K., Rosen, B.R., Polimeni, J.R., 2016a. Fast fMRI can detect oscillatory neural activity in humans. Proc. Natl. Acad. Sci. USA 113, E6679-E6685. http://dx.doi.org/10.1073/pnas.1608117113.

Lewis, L.D., Setsompop, K., Rosen, B.R., Polimeni, J.R., 2016b. Fast fMRI can detect oscillatory neural activity in humans. Proc. Natl. Acad. Sci. USA 113, E6679-E6685. http://dx.doi.org/10.1073/pnas.1608117113.

Liu, T., Spincemaille, P., De Rochefort, L., Kressler, B., Wang, Y., 2009. Calculation of susceptibility through multiple orientation sampling (COSMOS): a method for conditioning the inverse problem from measured magnetic field map to susceptibility source image in MRI. Magn. Reson. Med. 61, 196-204. http://dx.doi.org/10.1002/ mrm.21828.

Liu, T., Liu, J., de Rochefort, L., Spincemaille, P., Khalidov, I., Ledoux, J.R., Wang, Y., 2011. Morphology enabled dipole inversion (MEDI) from a single-angle acquisition: comparison with COSMOS in human brain imaging. Magn. Reson. Med. 66, $777-783$.

Lu, H., van Zijl, P.C.M., 2012. A review of the development of Vascular-Space-Occupancy (VASO) fMRI. Neuroimage. http://dx.doi.org/10.1016/j.neuroimage.2012.01.013.

Lu, H., Golay, X., Pekar, J.J., Van Zijl, P.C.M., 2003. Functional magnetic resonance imaging based on changes in vascular space occupancy. Magn. Reson. Med. 50, 263-274. http://dx.doi.org/10.1002/mrm.10519.

Lu, H., Van Zijl, P.C.M., Hendrikse, J., Golay, X., 2004. Multiple acquisitions with global inversion cycling (MAGIC): a multislice technique for vascular-space-occupancy dependent fMRI. Magn. Reson. Med. 51, 9-15. http://dx.doi.org/10.1002/ mrm.10659.

Lu, H., Hua, J., van Zijl, P.C.M., 2013. Noninvasive functional imaging of cerebral blood volume with vascular-space-occupancy (VASO) MRI. NMR Biomed. 26, 932-948. http://dx.doi.org/10.1002/nbm.2905.

Luh, W.-M., Talagala, S.L., Li, T.-Q., Bandettini, P. a, 2013. Pseudo-continuous arterial spin labeling at $7 \mathrm{~T}$ for human brain: estimation and correction for off-resonance effects using a Prescan. Magn. Reson. Med. 69, 402-410. http://dx.doi.org/10.1002/ mrm. 24266 .

Ma, C., Lam, F., Ning, Q., Johnson, C.L., Liang, Z.-P., 2016. High-resolution (1) H-MRSI of the brain using short-TE SPICE. Magn. Reson. Med.. http://dx.doi.org/10.1002/ mrm.26130.

Ma, D., Gulani, V., Seiberlich, N., Liu, K., Sunshine, J.L., Duerk, J.L., Griswold, M.A., 2013. Magnetic resonance fingerprinting. Nature 495, 187-192. http://dx.doi.org/ 10.1038/nature11971.

Malik, S.J., Keihaninejad, S., Hammers, A., Hajnal, J.V., 2012. Tailored excitation in 3D with spiral nonselective (SPINS) RF pulses. Magn. Reson. Med. 67, 1303-1315. http://dx.doi.org/10.1002/mrm.23118.

Mandeville, J.B., Marota, J.J.A., Ayata, C., Zaharchuk, G., Moskowitz, M.A., Rosen, B.R., Weisskoff, R.M., 1999. Evidence of a Cerebrovascular Postarteriole Windkessel With Delayed Compliance. J. Cereb. Blood Flow Metab. 19, 679-689. http://dx.doi.org/ 10.1097/00004647-199906000-00012.

Marques, J.P., Bowtell, R., 2005. Application of a Fourier-based method for rapid calculation of field inhomogeneity due to spatial variation of magnetic susceptibility. Concepts Magn. Reson. Part B Magn. Reson. Eng. 25B, 65-78.

Marques, J.P., Kober, T., Krueger, G., van der Zwaag, W., Van de Moortele, P.-F., Gruetter, R., 2010. MP2RAGE, a self bias-field corrected sequence for improved segmentation and T1-mapping at high field. Neuroimage 49, 1271-1281. http:// dx.doi.org/10.1016/j.neuroimage.2009.10.002.

Menon, R.S., 2002. Postacquisition suppression of large-vessel BOLD signals in highresolution fMRI. Magn. Reson. Med. 47, 1-9.

Menon, R.S., Goodyear, B.G., 1999. Submillimeter functional localization in human striate cortex using BOLD contrast at $4 \mathrm{~T}$ : implications for the vascular point-spread function. Magn. Reson. Med. 41, 230-235.

Miller, K.L., Smith, S.M., Jezzard, P., Pauly, J.M., 2006. High-resolution FMRI at 1.5 T using balanced SSFP. Magn. Reson. Med. 55, 161-170. http://dx.doi.org/10.1002/ mrm.20753.

Moeller, S., Yacoub, E., Olman, C. a, Auerbach, E.J., Strupp, J., Harel, N., Uğurbil, K., 2010. Multiband multislice GE-EPI at $7 \mathrm{~T}$, with 16-fold acceleration using partial parallel imaging with application to high spatial and temporal whole-brain fMRI. Magn. Reson. Med. 63, 1144-1153. http://dx.doi.org/10.1002/mrm.22361.

Mooiweer, R., Sbrizzi, A., Raaijmakers, A.J.E., van den Berg, C.A.T., Luijten, P.R., Hoogduin, H., 2016. Combining a reduced field of excitation with SENSE-based parallel imaging for maximum imaging efficiency. Magn. Reson. Med.. http:// dx.doi.org/10.1002/mrm.26346.

Moriguchi, H., Duerk, J.L., 2006. Bunched phase encoding (BPE): a new fast data acquisition method in MRI. Magn. Reson. Med. 55, 633-648. http://dx.doi.org/ 10.1002/mrm.20819.

Mugler, J.P., Brookeman, J.R., 1990. Three-dimensional magnetization-prepared rapid gradient-echo imaging (3D MP RAGE). Magn. Reson. Med. 15, 152-157.

Mugler, J.P., Epstein, F.H., Brookeman, J.R., 1992. Shaping the signal response during the approach to steady state in three-dimensional magnetization-prepared rapid gradient-echo imaging using variable flip angles. Magn. Reson. Med. 28, 165-185.

Narsude, M., Gallichan, D., Van Der Zwaag, W., Gruetter, R., Marques, J.P., 2016. Threedimensional echo planar imaging with controlled aliasing: a sequence for high temporal resolution functional MRI. Magn. Reson. Med. 75, 2350-2361. http:// dx.doi.org/10.1002/mrm.25835.

Norris, D.G., 2006. Principles of magnetic resonance assessment of brain function. J. Magn. Reson. Imaging 23, 794-807. http://dx.doi.org/10.1002/jmri.20587.

Norris, D.G., Koopmans, P.J., Boyacioğlu, R., Barth, M., 2011. Power Independent of Number of Slices (PINS) radiofrequency pulses for low-power simultaneous multislice excitation. Magn. Reson. Med. 66, 1234-1240. http://dx.doi.org/ $10.1002 / \mathrm{mrm} .23152$.

Norris, D.G., Boyacioğlu, R., Schulz, J., Barth, M., Koopmans, P.J., 2014. Application of PINS radiofrequency pulses to reduce power deposition in RARE/turbo spin echo 
imaging of the human head. Magn. Reson. Med. 71, 44-49. http://dx.doi.org/ 10.1002/mrm.24991.

Nunes, R.G., Hajnal, J.V., Golay, X., Larkman, D.J., 2006. Simultaneous slice excitation and reconstruction for single shot EPI., In: Proceedings International Soc Mag Reson Med. p. 293.

O’Herron, P., Chhatbar, P.Y., Levy, M., Shen, Z., Schramm, A.E., Lu, Z., Kara, P., 2016. Neural correlates of single-vessel haemodynamic responses in vivo. Nature 534 378-382. http://dx.doi.org/10.1038/nature17965.

Ohliger, M.A., Grant, A.K., Sodickson, D.K., 2003. Ultimate intrinsic signal-to-noise ratio for parallel MRI: electromagnetic field considerations. Magn. Reson. Med. 50, 1018-1030. http://dx.doi.org/10.1002/mrm.10597.

Olman, C.A., Harel, N., Feinberg, D.A., He, S., Zhang, P., Ugurbil, K., Yacoub, E., 2012. Layer-specific fMRI reflects different neuronal computations at different depths in human V1. PLoS One 7, e32536. http://dx.doi.org/10.1371/journal.pone.0032536.

Oshio, K., Feinberg, D.A., 1991. GRASE (Gradient- and spin-echo) imaging: a novel fast MRI technique. Magn. Reson. Med. 20, 344-349.

Pfeuffer, J., Adriany, G., Shmuel, A., Yacoub, E., Van De Moortele, P.-F., Hu, X., Ugurbil, K., 2002a. Perfusion-based high-resolution functional imaging in the human brain at 7 T. Magn. Reson. Med. 47, 903-911. http://dx.doi.org/10.1002/mrm.10154.

Pfeuffer, J., Van de Moortele, P.-F., Yacoub, E., Shmuel, A., Adriany, G., Andersen, P., Merkle, H., Garwood, M., Uğurbil, K., Hu, X., 2002b. Zoomed functional imaging in the human brain at $7 \mathrm{~T}$ with simultaneous high spatial and high temporal resolution. Neuroimage 17, 272-286. http://dx.doi.org/10.1006/nimg.2002.1103.

Polimeni, J.R., Fischl, B., Greve, D.N., Wald, L.L., 2010. Laminar analysis of 7T BOLD using an imposed spatial activation pattern in human V1. Neuroimage 52, 1334-1346. http://dx.doi.org/10.1016/j.neuroimage.2010.05.005.

Polimeni, J.R., Bhat, H., Witzel, T., Benner, T., Feiweier, T., Inati, S.J., Renvall, V., Heberlein, K., Wald, L.L., 2016. Reducing sensitivity losses due to respiration and motion in accelerated echo planar imaging by reordering the autocalibration data acquisition. Magn. Reson. Med. 75, 665-679. http://dx.doi.org/10.1002/ mrm. 25628 .

Poser, B., Ivanov, D., Barth, M., Uludağ, K., 2015. High resolution 3D EPI at 7 and 9.4T and its application to quantitative susceptibility mapping. In: OHBM. p. 1577.

Poser, B., Kaas, A., Wiggins, C., Uludag, K., Tse, D., 2017. Dual region-selective spiral pTX excitation for digit mapping fMRI in motor cortex and cerebellum. In: Proceedings International Soc Mag Reson Med.

Poser, B.A., Norris, D.G., 2009. 3D single-shot VASO using a maxwell gradient compensated GRASE sequence. Magn. Reson. Med. 62. http://dx.doi.org/10.1002/ mrm.22000.

Poser, B.A., Norris, D.G., 2011. Application of whole-brain CBV-weighted fMRI to a cognitive stimulation paradigm: robust activation detection in a stroop task experiment using 3D GRASE VASO. Hum. Brain Mapp. 32, 974-981. http:// dx.doi.org/10.1002/hbm.21083.

Poser, B.A., Tse, D.H.Y., 2015. 2D EPI at 9.4T with slice-specific spokes pulse RF excitation for B1+ homogenisation. In: ISMRM. p. 593.

Poser, B.A., Koopmans, P.J., Witzel, T., Wald, L.L., Barth, M., 2010. Three dimensional echo-planar imaging at 7 T. Neuroimage 51. http://dx.doi.org/10.1016/ j.neuroimage.2010.01.108.

Poser, B.A., Anderson, R.J., Guérin, B., Setsompop, K., Deng, W., Mareyam, A., Serano, P., Wald, L.L., Stenger, V.A., 2014. Simultaneous multislice excitation by parallel transmission. Magn. Reson. Med. 71. http://dx.doi.org/10.1002/mrm.24791.

Pruessmann, K.P., Weiger, M., Scheidegger, M.B., Boesiger, P., 1999. SENSE: sensitivity encoding for fast MRI. Magn. Reson. Med. 42, 952-962.

de Rochefort, L., Liu, T., Kressler, B., Liu, J., Spincemaille, P., Lebon, V., Wu, J., Wang, Y., 2010. Quantitative susceptibility map reconstruction from MR phase data using bayesian regularization: validation and application to brain imaging. Magn. Reson. Med. 63, 194-206. http://dx.doi.org/10.1002/mrm.22187.

Saekho, S., Yip, C., Noll, D.C., Boada, F.E., Stenger, V.A., 2006. Fast-kz threedimensional tailored radiofrequency pulse for reduced B1 inhomogeneity. Magn. Reson. Med. 55, 719-724. http://dx.doi.org/10.1002/mrm.20840.

Satpute, A.B., Wager, T.D., Cohen-Adad, J., Bianciardi, M., Choi, J.-K., Buhle, J.T., Wald, L.L., Barrett, L.F., 2013. Identification of discrete functional subregions of the human periaqueductal gray. Proc. Natl. Acad. Sci. USA 110, 17101-17106. http:// dx.doi.org/10.1073/pnas.1306095110.

Sbrizzi, A., Poser, B.A., Tse, D.H.Y., Hoogduin, H., Luijten, P.R., van den Berg, C.A.T., 2015. RF peak power reduction in CAIPIRINHA excitation by interslice phase optimization. NMR Biomed. 28. http://dx.doi.org/10.1002/nbm.3360.

Scheffler, K., Ehses, P., 2016. High-resolution mapping of neuronal activation with balanced SSFP at 9.4 T. Magn. Reson. Med. 76, 163-171. http://dx.doi.org/ $10.1002 / \mathrm{mrm} .25890$

Scheffler, K., Seifritz, E., Bilecen, D., Venkatesan, R., Hennig, J., Deimling, M., Haacke, E.M., 2001. Detection of BOLD changes by means of a frequency-sensitive trueFISP technique: preliminary results. NMR Biomed. 14, 490-496. http://dx.doi.org/ $10.1002 / \mathrm{nbm} .726$.

Schulz, J., Boyaciołlu, R., Norris, D.G., 2016. Multiband multislab 3D time-of-flight magnetic resonance angiography for reduced acquisition time and improved sensitivity. Magn. Reson. Med. 75, 1662-1668. http://dx.doi.org/10.1002/ mrm. 25774.

Setsompop, K., Alagappan, V., Gagoski, B., Witzel, T., Polimeni, J.R., Potthast, A., Hebrank, F., Fontius, U., Schmitt, F., Wald, L.L., Adalsteinsson, E., 2008. Sliceselective RF pulses for in vivo B1+ inhomogeneity mitigation at $7 \mathrm{~T}$ using parallel RF excitation with a 16-element coil. Magn. Reson. Med. 60, 1422-1432. http:// dx.doi.org/10.1002/mrm.21739.

Setsompop, K., Gagoski, B.A.B., Polimeni, J.R., Witzel, T., Wedeen, V.J., Wald, L.L., 2012. Blipped-controlled aliasing in parallel imaging for simultaneous multislice echo planar imaging with reduced g-factor penalty. Magn. Reson. Med. 67,
1210-1224. http://dx.doi.org/10.1002/mrm.23097.

Setsompop, K., Feinberg, D.A., Polimeni, J.R., 2016. Rapid brain MRI acquisition techniques at ultra-high fields. NMR Biomed.. http://dx.doi.org/10.1002/nbm.3478.

Sharma, A., Holdsworth, S.J., O’Halloran, R., Aboussouan, E., Van, A.T., Maclaren, J., Aksoy, M., Stenger, A.V., Bammer, R., Grissom, W.A., 2013. kT-PINS RF Pulses for Low-Power Field Inhomogeneity-Compensated Multislice Excitation, In: Proceedings International Soc. Mag. Reson. Med. p. 73.

Shmueli, K., Zwart, J. De, 2009. Magnetic susceptibility mapping of brain tissue in vivo using MRI phase data. Magn. Reson. Med. 62, 1510-1511.

Siero, J.C.W., Petridou, N., Hoogduin, H., Luijten, P.R., Ramsey, N.F., 2011. Cortical depth-dependent temporal dynamics of the BOLD response in the human brain. J. Cereb. Blood Flow Metab. 31, 1999-2008. http://dx.doi.org/10.1038/ jcbfm.2011.57.

Talagala, S.L., Sarlls, J.E., Inati, S.J., 2013. Improved temporal SNR of accelerated EPI using a FLASH based GRAPPA reference scan. Proc. Int. Soc. Mag. Reson. Med. 21, 2658.

Tamir, J.I., Uecker, M., Chen, W., Lai, P., Alley, M.T., Vasanawala, S.S., Lustig, M., 2016. T2 shuffling: sharp, multicontrast, volumetric fast spin-echo imaging. Magn. Reson. Med.. http://dx.doi.org/10.1002/mrm.26102.

Teeuwisse, W.M., Webb, A.G., Van Osch, M.J.P., 2010. Arterial spin labeling at ultrahigh field: all that glitters is not gold. Int. J. Imaging Syst. Technol. 20, 62-70. http://dx.doi.org/10.1002/ima.20218.

Tian, L., Kong, Y., Ren, J., Varoquaux, G., Zang, Y., Smith, S.M., 2013. Spatial vs. temporal features in ICA of resting-state fMRI - a quantitative and qualitative investigation in the context of response inhibition. PLoS One 8, e66572. http:// dx.doi.org/10.1371/journal.pone.0066572.

Trampel, R., Bazin, P.-L., Schäfer, A., Heidemann, R.M., Ivanov, D., Lohmann, G., Geyer, S., Turner, R., 2012. Laminar-specific fingerprints of different sensorimotor areas obtained during imagined and actual finger tapping. Proc. Int. Soc. Mag. Reson. Med. 20, 663.

Tse, Wiggins, P., 2016b. Estimating and eliminating the cause of excitation errors in bipolar gradient composite excitations: example of bipolar spokes pulses in parallel transmission. Magn. Reson. Med. (accepted), MRM-16-17304-R2.

Tse, Wiggins, Poser, 2016a. High-resolution gradient-recalled echo imaging at 9.4T using 16-channel parallel transmit simultaneous multislice spokes excitations with sliceby-slice flip angle homogenization. Magn. Reson. Med.. http://dx.doi.org/10.1002/ mrm.26501.

Uğurbil, K., Xu, J., Auerbach, E.J., Moeller, S., Vu, A.T., Duarte-Carvajalino, J.M., Lenglet, C., Wu, X., Schmitter, S., Van de Moortele, P.F., Strupp, J., Sapiro, G., De Martino, F., Wang, D., Harel, N., Garwood, M., Chen, L., Feinberg, D.A., Smith, S.M., Miller, K.L., Sotiropoulos, S.N., Jbabdi, S., Andersson, J.L.R., Behrens, T.E.J., Glasser, M.F., Van Essen, D.C., Yacoub, E., 2013. Pushing spatial and temporal resolution for functional and diffusion MRI in the Human Connectome Project. Neuroimage 80, 80-104. http://dx.doi.org/10.1016/j.neuroimage.2013.05.012.

von Morze, C., Xu, D., Purcell, D.D., Hess, C.P., Mukherjee, P., Saloner, D., Kelley, D.A.C., Vigneron, D.B., 2007. Intracranial time-of-flight MR angiography at 7T with comparison to 3T. J. Magn. Reson. Imaging 26, 900-904. http://dx.doi.org/ 10.1002/jmri.21097.

von Morze, C., Kelley, D. a C., Shepherd, T.M., Banerjee, S., Xu, D., Hess, C.P., 2010 Reduced field-of-view diffusion-weighted imaging of the brain at 7 T. Magn. Reson. Imaging 28, 1541-1545. http://dx.doi.org/10.1016/j.mri.2010.06.025.

Vu, A.T., Feinberg, D.A., Harel, N., Uğurbil, K., Yacoub, E.S., 2013. Diagonal multi-slab inner volume 3D GRASE imaging for high resolution T2 weighted fMRI. Proc. Int. Soc. Mag. Reson. Med. 21.

Vu, A.T., Auerbach, E., Lenglet, C., Moeller, S., Sein, J., Moortele, De, P.V., Ugurbil, K. Yacoub, E., 2014. . High resolution whole brain diffusion imagining at 7T for the Human Connectome Project. In: Proceedings International Soc Mag Reson Med. p. 1000.

Vu, A.T., Auerbach, E., Lenglet, C., Moeller, S., Sotiropoulos, S.N., Jbabdi, S., Andersson, J., Yacoub, E., Ugurbil, K., 2015. High resolution whole brain diffusion imaging at 7T for the Human Connectome Project. Neuroimage 122, 318-331. http://dx.doi.org/ 10.1016/j.neuroimage.2015.08.004.

Vu, A.T., Jamison, K., Glasser, M.F., Smith, S.M., Coalson, T., Moeller, S., Auerbach, E.J., Uğurbil, K., Yacoub, E., 2016. Tradeoffs in pushing the spatial resolution of fMRI for the 7T Human Connectome Project. Neuroimage.. http://dx.doi.org/10.1016/ j.neuroimage.2016.11.049.

Wiesinger, F., de Moortele, Van, Adriany, P.-F., De Zanche, G., Ugurbil, N., Pruessmann, K.P, K., 2004. Parallel imaging performance as a function of field strength-an experimental investigation using electrodynamic scaling. Magn. Reson. Med. 52, 953-964. http://dx.doi.org/10.1002/mrm.20281.

Wiesinger, F., Zanche, N. De, Pruessmann, K.P., 2005. . Approaching Ultimate SNR with Finite Coil Arrays. In: Proceedings International Soc. Mag. Reson. Med. p. 672.

Wiesinger, F., de Moortele, Van, Adriany, P.F., De Zanche, G., Ugurbil, N., Pruessmann, K.P, K., 2006. Potential and feasibility of parallel MRI at high field. NMR Biomed. 19, 368-378. http://dx.doi.org/10.1002/nbm.1050.

Wiggins, G.C., Triantafyllou, C., Potthast, A., Reykowski, A., Nittka, M., Wald, L.L., 2006. 32-Channel $3 \mathrm{~T}$ receive-only phased-array head coil with soccer-ball element geometry. Magn. Reson. Med. 56, 216-223. http://dx.doi.org/10.1002/mrm.20925.

Wiggins, G.C., Polimeni, J.R., Potthast, A., Schmitt, M., Alagappan, V., Wald, L.L., 2009. 96-Channel receive-only head coil for $3 \mathrm{~T}$ : design optimization and evaluation. Magn. Reson. Med. 62, 754-762. http://dx.doi.org/10.1002/mrm.22028.

Williams, D.S., Detre, J.A., Leigh, J.S., Koretsky, A.P., 1992. Magnetic resonance imaging of perfusion using spin inversion of arterial water. Proc. Natl. Acad. Sci. USA 89, 212-216. http://dx.doi.org/10.1073/pnas.89.9.4220e.

Wu, W., Poser, B.A., Douaud, G., Frost, R., In, M.-H., Speck, O., Koopmans, P.J., Miller, K.L., 2016a. High-resolution diffusion MRI at 7T using a three-dimensional multi- 
slab acquisition. Neuroimage 143, 1-14. http://dx.doi.org/10.1016/ j.neuroimage.2016.08.054.

Wu, X., Schmitter, S., Auerbach, E.J., Moeller, S., Uğurbil, K., Van De Moortele, P.F., 2013. Simultaneous multislice multiband parallel radiofrequency excitation with independent slice-specific transmit B1 homogenization. Magn. Reson. Med. 70, 630-638. http://dx.doi.org/10.1002/mrm.24828.

Wu, X., Schmitter, S., Auerbach, E.J., Uıurbil, K., Van De Moortele, P.F., 2016b. A generalized slab-wise framework for parallel transmit multiband RF pulse design. Magn. Reson. Med. 75, 1444-1456. http://dx.doi.org/10.1002/mrm.25689.

Xu, J., Moeller, S., Auerbach, E.J., Strupp, J., Smith, S.M., Feinberg, D.A., Yacoub, E., Uğurbil, K., 2013. Evaluation of slice accelerations using multiband echo planar imaging at 3T. Neuroimage 83, 991-1001. http://dx.doi.org/10.1016/ j.neuroimage.2013.07.055.

Yacoub, E., Shmuel, A., Pfeuffer, J., Van De Moortele, P.F., Adriany, G., Andersen, P., Vaughan, J.T., Merkle, H., Ugurbil, K., Hu, X., 2001. Imaging brain function in humans at 7 T. Magn. Reson. Med. 45, 588-594.

Yacoub, E., Van De Moortele, P.F., Shmuel, A., Uğurbil, K., 2005. Signal and noise characteristics of Hahn SE and GE BOLD fMRI at 7 T in humans. Neuroimage 24, 738-750. http://dx.doi.org/10.1016/j.neuroimage.2004.09.002.

Yacoub, E., Harel, N., Ugurbil, K., 2008. High-field fMRI unveils orientation columns in humans. Proc. Natl. Acad. Sci. USA 105, 10607-10612. http://dx.doi.org/10.1073/ pnas.0804110105.
Ye, H., Ma, D., Jiang, Y., Cauley, S.F., Du, Y., Wald, L.L., Griswold, M. a, Setsompop, K., 2015. Accelerating magnetic resonance fingerprinting (MRF) using t-blipped simultaneous multislice (SMS) acquisition. Magn. Reson. Med. Earlyview. http:// dx.doi.org/10.1002/mrm.25799.

Ye, H., Cauley, S.F., Gagoski, B., Bilgic, B., Ma, D., Jiang, Y., Du, Y.P., Griswold, M.A., Wald, L.L., Setsompop, K., 2016. Simultaneous multislice magnetic resonance fingerprinting (SMS-MRF) with direct-spiral slice-GRAPPA (ds-SG) reconstruction Magn. Reson. Med., 0. http://dx.doi.org/10.1002/mrm.26271.

Zahneisen, B., Poser, B. a, Ernst, T., Stenger, V.A., 2014. Three-dimensional Fourier encoding of simultaneously excited slices: generalized acquisition and reconstruction framework. Magn. Reson. Med. 71, 2071-2081. http://dx.doi.org/10.1002/ mrm. 24875 .

Zahneisen, B., Ernst, T., Poser, B. a, 2015. SENSE and simultaneous multislice imaging. Magn. Reson. Med.. http://dx.doi.org/10.1002/mrm.25519, (doi:10.1002/ mrm.25519).

Zuo, Z., Wang, R., Zhuo, Y., Xue, R., St Lawrence, K.S., Wang, D.J.J., 2013. TurboFLASH based arterial spin labeled perfusion MRI at 7 T. PLoS One 8, e66612. http://dx.doi.org/10.1371/journal.pone.0066612.

Zwanenburg, J.J.M., Hendrikse, J., Takahara, T., Visser, F., Luijten, P.R., 2008. MR angiography of the cerebral perforating arteries with magnetization prepared anatomical reference at 7 T: comparison with time-of-flight. J. Magn. Reson. Imaging 28, 1519-1526. http://dx.doi.org/10.1002/jmri.21591. 